\title{
ON THE PRESENT STATE OF THE ANDERSÉN-LEMPERT THEORY
}

\author{
SHULIM KALIMAN AND FRANK KUTZSCHEBAUCH
}

Dedicated to Professor Peter Russell

on the occasion of his seventieth birthday

\begin{abstract}
In this survey of the AnDERSÉn-LEMPERT theory we present the state of the art in the study of the density property (which means that the Lie algebra generated by completely integrable holomorphic vector fields on a given Stein manifold is dense in the space of all holomorphic vector fields). There are also two new results in the paper one of which is the theorem stating that the product of Stein manifolds with the volume density property possesses such a property as well. The second one is a meaningful example of an algebraic surface without the algebraic density property. The proof of the last fact requires BRUNELLA's technique.
\end{abstract}

\section{INTRODUCTION}

In this paper we discuss recent developments in the ANDERSÉN-LEMPERT theory. This theory describes complex manifolds such that among other things the local phase flows on their holomorphically convex compact subsets can be approximated by global holomorphic automorphisms which leads to construction of holomorphic automorphisms with prescribed local properties. Needless to say that this implies remarkable consequences for such manifolds some of which are described below in Section 2. The original work of Andersén and Lempert ([2], 3]) established that complex Euclidean spaces of dimension at least 2 belong to this class. Their results were extended by Forstnerič and Rosay [29], [57] who discovered new approximation theorems for such spaces. Perhaps, they understood already that a complex manifold has such approximations if it possesses the following density property introduced later by VAROLIN.

1.1. Definition. A complex manifold $X$ has the density property if in the compact-open topology the Lie algebra $\operatorname{Lie}_{\text {hol }}(X)$ generated by completely integrable holomorphic vector fields on $X$ is dense in the Lie algebra $\operatorname{VF}_{\text {hol }}(X)$ of all holomorphic vector fields on $X$. An affine algebraic manifold $X$ has the algebraic density property if the Lie algebra $\operatorname{Lie}_{\text {alg }}(X)$ generated by completely integrable algebraic vector fields on it coincides with the Lie algebra $\mathrm{VF}_{\text {alg }}(X)$ of all algebraic vector fields on it.

2000 Mathematics Subject Classification. Primary: 32M05,14R20. Secondary: 14R10, 32M25. 
Usually the easiest way to establish the density property for an affine algebraic manifold $X$ is to prove the algebraic density property for it since some convenient tools from affine algebraic geometry are available in this cas $\ell^{1}$. In the sequence of papers ([70], 67], 68], 44], 45], [21]) the algebraic density property was established for a wide variety of affine algebraic manifolds, including all homogeneous spaces that coincide with quotients of linear algebraic groups with respect to their reductive subgroups provided that the connected components of these quotients are different from $\mathbb{C}$ or complex tori. The last two papers were based on a very effective criterion of the algebraic density property introduced by the authors which will be presented in Section 3 .

Andersén, Lempert, Forstnerič, Rosay, Toth, VArolin, and the authors considered also another property which has similar consequences for automorphisms preserving a volume form.

1.2. Definition. Let a complex manifold $X$ be equipped with a holomorphic volume form $\omega$ (i.e. $\omega$ is nowhere vanishing section of the canonical bundle). We say that $X$ has the volume density property with respect to $\omega$ if in the compact-open topology the Lie algebra Lie $\omega_{\text {hol }}^{\omega}$ generated by completly integrable holomorphic vector fields $\nu$ such that $\nu(\omega)=0$, is dense in the Lie algebra $\operatorname{VF}_{\text {hol }}^{\omega}(X)$ of all holomorphic vector fields that annihilate $\omega$ (note that condition $\nu(\omega)=0$ is equivalent to the fact that $\nu$ is of $\omega$-divergence zero). If $X$ is affine algebraic we say that $X$ has the algebraic volume density property with respect to an algebraic volume form $\omega$ if the Lie algebra $\mathrm{Lie}_{\text {alg }}^{\omega}$ generated by completely integrable algebraic vector fields $\nu$ such that $\nu(\omega)=0$, coincides with the Lie algebra $\operatorname{VF}_{\text {alg }}^{\omega}(X)$ of all algebraic vector fields that annihilate $\omega$.

It is much more difficult to establish the algebraic volume density property than the algebraic density property since the criterion mentioned before is not applicable in the volume case. However ANDERSÉn [2] established the algebraic volume density property for Euclidean spaces even before the algebraic density property. Some extra manifolds with the algebraic volume density were found by VAROLIN and eventually the authors [45] proved it for all linear algebraic groups (with respect to the left or right invariant volume forms). Furthermore, they established some features that are straightforward for the algebraic density property and not at all clear in the volumepreserving case. For instance, they prove that the algebraic volume density property for an affine algebraic manifold $X$ implies the volume density property for such an $X$ and that the product of two manifolds with algebraic volume density property has again the algebraic volume density property. Some facts on this subject are contained in Section 4 together with the following new result.

Theorem 1. Let $X$ and $Y$ be Stein manifolds equipped with (holomorphic) volume forms $\omega_{X}$ and $\omega_{Y}$ respectively. Suppose that $X$ and $Y$ have the volume density property with respect to these forms. Then so does $X \times Y$ with respect to the form $\omega_{X} \times \omega_{Y}$.

\footnotetext{
${ }^{1}$ Furthermore, the authors do not know examples of such an $X$ with the density property but without the algebraic density property.
} 
Unlike in the algebraic case the proof of this theorem required some nontrivial facts from functional analysis.

In Sections 5-8 we consider a new meaningful example of an affine surface without the algebraic density property. The algebraic density property for an affine algebraic algebraic manifold implies that this manifold has an $m$-transitive group of holomorphic automorphisms for any natural $m$ (i.e. every $m$-tuple of distinct points in the manifold can be transformed into any other such tuple by an automorphism). Thus it makes sense to consider such objects only. The question about examples of $m$-transitive affine algebraic manifolds (for any $m$ ) without the algebraic density property were posed by P. Russell and D. Akhiezer to the first author who was not aware at the time about the third paper of ANDERSÉn [4] showing that tori belong to this class. However his proof is based heavily on a Borel theorem from the Nevanlinna theory which is quite specific for tori. The question whether there are examples besides tori and the line was still open.

We shall show that the surface $S$ given by $x+y+x y z=1$ in $\mathbb{C}^{3}$ does not have the algebraic density property. The proof will be based on remarkable BRUNELLA's technique [10], [13], [12, that allowed him to classify completely integrable algebraic vector fields on the plane (which is based in turn on the seminal preprint of MCQUILLAN [54] and earlier work of Suzuki 65, 66]). In fact, his technique works for other rational affine surfaces (including the tori $\left(\mathbb{C}^{*}\right)^{2}$ ) and, therefore, is more productive than the ANDERSÉN's approach in the two dimensional case. We present crucial ingredients of his method in Section 5-7 and apply them to $S$ in Section 8.

Acknowledgements. It is a pleasure to express our gratitude to NAHUM ZoBIN for explaining us important facts from functional analysis.

\section{Applichtions of the Andersén-Lempert theory}

Constructions of holomorphic automorphisms of Stein manifolds with prescribed behavior on compact subsets are based on the next central theorem of the ANDERSÉNLEMPERT theory which was proven in the papers [2] and [3] of ANDERSÉn and LEMPERT for Euclidean spaces. We give a stronger version of this theorem which is due to Forstnerič and Rosay [29]. They considered it also in the case Euclidean spaces only; however it was essentially their contribution that made the original result an important tool as it is now.

Theorem 2. Let $X$ be a Stein manifold with the density (resp. volume density) property and let $\Omega$ be an open subset of $X$. Suppose that $\Phi:[0,1] \times \Omega \rightarrow X$ is a $C^{1}$-smooth map such that

(1) $\Phi_{t}: \Omega \rightarrow X$ is holomorphic and injective (and resp. volume preserving) for every $t \in[0,1]$

(2) $\Phi_{0}: \Omega \rightarrow X$ is the natural embedding of $\Omega$ into $X$, and 
(3) $\Phi_{t}(\Omega)$ is a Runge subse ${ }^{2}$ of $X$ for every $t \in[0,1]$.

Then for each $\varepsilon>0$ and every compact subset $K \subset \Omega$ there is a continuous family, $\alpha:[0,1] \rightarrow \operatorname{Aut}_{h o l}(X)$ of holomorphic (and resp. volume preserving) automorphisms of $X$ such that

for every $t \in[0,1]$.

$$
\alpha_{0}=\Phi_{0} \text { and }\left|\alpha_{t}-\Phi_{t}\right|_{K}<\varepsilon
$$

Furthermore, approximations on Stein manifolds with the density property can be chosen with some specific features as in the next result [71].

2.1. Proposition. Let $X$ be a Stein manifold of dimension $n \geq 2$ with the density (resp. volume density) property, $K$ be a compact in $X$, and $x, y \in X$ be two points outside the convex hull of $K$. Suppose that $x_{1}, \ldots, x_{m} \in K$.

Then there exists a (resp. volume-preserving) holomorphic automorphism $\Psi$ of $K$ such that $\Psi\left(x_{i}\right)=x_{i}$ for every $i=1, \ldots, m,\left.\Psi\right|_{K}: K \rightarrow X$ is as close to the natural embedding as we wish, and $\Psi(y)=x$.

2.2. Remark. This Proposition 2.1 was proven in [71] for the density property only but a slightly modified argument works in the volume-preserving case. More precisely, let $\gamma$ be a piece-wise analytic path between $y$ and $x$ that does not meet the holomorphic hull $K_{0}$ of $K, K_{1}$ be the union of $K_{0}$ and a small ball $U$ around $y$, and $\gamma_{1}=U \cap$ $\gamma$. The main step in construction of $\Psi$, where the adjustment is needed, is a global approximation on $X$ of a holomorphic vector field $\nu$ on $K_{1}$ which is identically zero on $K_{0}$ and which is tangent to $\gamma_{1}$. In the volume-preserving case not only $\nu$ but also its global approximation must be of divergence zero which prevents the direct use of the Runge property as in [71]. However, it turns out that the existence of such an approximation is equivalent to approximation of some closed holomorphic $(n-1)$-form $\alpha$ on $K_{1}$ that is identically zero on $K_{0}$ by a global closed holomorphic $(n-1)$-form on $X$ (see the proof of Claim in Lemma 4.1 for more accurate details). Note that $\alpha$ is exact (indeed it is zero on $K_{0}$ and $H^{n-1}(U, \mathbb{C})=0$ which enables us to apply de Rham's theorem), i.e. $\alpha=\mathrm{d} \beta$ where $\beta$ is a holomorphic $(n-2)$-form on $K_{1}$. Since $K_{1}$ is still holomorphically convex, the Runge property implies that one can approximate $\beta$ by a global holomorphic $(n-2)$-form $\beta^{\prime}$ which yields an approximation of $\alpha$ by the global closed $(n-1)$-form $\mathrm{d} \beta^{\prime}$. We want to emphasize that this reasoning uses the assumption that $n \geq 2$ which is essential for the volume-preserving case. For $n=1$, consider, for example, $X=\mathbb{C}^{*}$ which has the volume density property with respect to the volume form $\mathrm{d} z / z$ where $z$ is the coordinate on $\mathbb{C}^{*}$. However it does not satisfy this analogue of Proposition 2.1. By the same reason it has no properties (B)-(E) described below.

\footnotetext{
${ }^{2}$ Recall that an open subset $U$ of $X$ is Runge if any holomorphic function on $U$ can be approximated by global holomorphic functions on $X$ in the compact-open topology. Actually, by Cartan's Theorem A this definition implies more: for any coherent sheaf on $X$ its section over $U$ can be approximated by global sections.
} 
Treating $X$ as a Stein $n$-dimensional manifold in the rest of this section, let us describe some general properties of Stein manifolds with density (resp. volume density) property which follow directly from the density property and from subtle applications of Theorem 2 and Proposition 2.1.

(A) If $X$ has the density (resp. volume density) property, then there are finitely many completely integrable holomorphic vector fields (resp. of divergence zero) that span the tangent space at each point (see 44] and Lemma 4.1 below). Therefore $X$ admits a spray 3 , i.e. it is elliptic in the sense of Gromov which implies, in particular, the Oka-Grauert-Gromov principle for submersions over Stein spaces with fibers isomorphic to $X$.

To be more precise let us give the relevant definitions and results.

2.3. Definition. (a) A (dominating) spray on a complex manifold $X$ is a holomorphic vector bundle $\rho: E \rightarrow X$, together with a holomorphic map $s: E \rightarrow X$, such that $s$ is identical on the zero section $X \hookrightarrow E$, and for each $x \in X$ the induced differential map sends the fibre $E_{x}=\rho^{-1}(x)$ (which is viewed as a linear subspace of $T_{x} E$ ) surjectively onto $T_{x} X$.

(b) A fiber-dominating spray for a surjective submersion $h: Z \rightarrow W$ of complex spaces is a vector bundle $\rho: E \rightarrow Z$ together with a map $s: E \rightarrow Z$ identical on the zero section $Z \hookrightarrow E$ and such that $h \circ s=h \circ p$ and for every $z \in Z$ the induced differential map sends $E_{z}=\rho^{-1}(z)$ (which is viewed as a linear subspace of $T_{z} E$ ) surjectively onto the subspace of $T_{z} Z$ tangent to the fiber $h^{-1}(h(z))$.

(c) Let $h: Z \rightarrow W$ be a holomorphic submersion of Stein spaces, and $\operatorname{Cont}(W, Z)$ (resp. $\operatorname{Holo}(W, Z)$ ) be the set of continuous (resp. holomorphic) sections of $h$. Then $h$ satisfies the Oka-Grauert-Gromov principle if $\operatorname{Holo}(W, Z) \hookrightarrow \operatorname{Cont}(W, Z)$ is a weak homotopy equivalence. That is,

(i) each continuous section $f^{0}: W \rightarrow Z$ of $h$ can be deformed to a holomorphic section $f^{1}: W \rightarrow Z$, and

(ii) any two homotopic holomorphic sections are also homotopic through holomorphic sections.

Theorem. (Oka-Grauert-Gromov-principle for elliptic submersions [37], 30]) Suppose that $h: Z \rightarrow W$ is a holomorphic submersion of a complex space $Z$ onto a Stein space $W$ for which every $x \in W$ has a neighborhood $U \subset W$ such that $h^{-1}(U) \rightarrow U$ admits a fiber-dominating spray. Then $h: Z \rightarrow W$ satisfies the Oka-Grauert-Gromov principle.

To illustrate this principle recall the following result of the authors [44].

\footnotetext{
${ }^{3}$ There is a small inaccuracy in 44 where the authors proved the existence of a spray. Namely, the metric on the space of holomorphic automorphisms of $X$ should be defined not as suggested in that paper but by formula (4.1) below.
} 
Theorem 3. The density (resp. algebraic density) property holds for smooth analytic (resp. algebraic) hypersurfaces in $\mathbb{C}_{u, v, \bar{x}}^{n+2}$ given by equations of form uv $=p(\bar{x})$.

Hence the Oka-Grauert-Gromov principle for submersions implies the following.

2.4. Corollary. Let $h: Z \rightarrow W$ be a surjective submersion of complex manifolds such that $W$ is Stein and for every $w_{0} \in W$ there is a neighborhood $U$ for which $h^{-1}(U)$ is naturally isomorphic to a hypersurface in $\mathbb{C}_{\bar{x}, u, v}^{n+2} \times U$ given by uv $=p(\bar{x}, w)$ where $p$ is a holomorphic function on $\mathbb{C}_{\bar{x}}^{n} \times U$ (independent of $u$ and $v$ ).

Suppose, furthermore, that $p^{*}(0) \cap\left(\mathbb{C}_{\bar{x}}^{n} \times w\right)$ is a smooth reduced proper (may be empty) submanifold of $\mathbb{C}_{\bar{x}}^{n} \times w$ for every point $w \in U$.

Then $h$ satisfies the Oka-Grauert-Gromov principle.

(B) If $X$ has the density property (or the volume density property) and the dimension $n$ of $X$ is at least 2, then the holomorphic automorphisms group $\operatorname{Authol}_{\text {hol }}(X)$ acts $m$-transitively on $X$ for any natural number $m$. This was mentioned by VAROLIN [71] for the density property as a simple consequence of Proposition 2.1, For the volume density property this works also due to Remark 2.2 .

(C) If $X$ has the density property (or the volume density property) and the dimension $n \geq 2$ then for each point $x \in X$ there is an injective but not surjective holomorphic map $f: X \rightarrow X$ with $f(x)=x$. The images of such maps are called Fatou-Bieberbach-domains of the second kind. This was also observed by VAROLIN in [71] for the density property, for the volume density property this is an equally simple application of the kick-out method of Dixon and EsTERLE [20].

(D) If $X$ has the density property, then for each point $x \in X$ there is an injective non-surjective equidimensional holomorphic map $f: \mathbb{C}^{n} \rightarrow X$ with $f(0)=x$. This observation is due to VAROLIN [71]. In particular, all Eisenman measures on $X$ vanish identically. Such maps are called Fatou-Bieberbach maps of the first kind and their images are Fatou-Bieberbach domains.

2.5. Remark. Here is a sketch of the proof of the last fact. Take a holomorphically convex neighborhood $\Omega$ of $x$ together with a vector field $\theta$ on $\Omega$ for which $x$ is an attractive point. Approximating the flow of this field by automorphisms of $X$ as in Theorem 2 we obtain an automorphism whose restriction to a neighborhood of $x$ is a contraction (in some metric) to a point near $x$. The basin of attraction of $x$ for that automorphism will be biholomorphic to $\mathbb{C}^{n}$. Clearly $\theta$ cannot be volume preserving when contracting to a point. Therefore the volume density property does not guarantee the existence of Fatou-Bieberbach domains. Say, it is known that $\left(\mathbb{C}^{*}\right)^{n}$ has the volume density property but for $n \geq 2$ it is an open question whether it contains a FatouBieberbach domain or not. In particular, it is unknown whether it has the density property as well. 
(E) If $X$ has the density (resp. volume density) property and $Y$ is any manifold which admits a proper holomorphic embedding $\varphi: Y \hookrightarrow X$ then the following is true. For any given discrete subset $E=\left\{x_{1}, x_{2}, \ldots, x_{m}, \ldots\right\}$ in $X$ there is another proper holomorphic embedding $\psi$ of $Y$ into $X$ whose image contains $E$. The original proof of this fact was obtained by Globevnik, Forstnerič and Rosay [34] in the case of $X=\mathbb{C}^{n}, n \geq 2$ but it works also in the general case. This proof is based on Proposition 2.1. Namely, if the image of a proper holomorphic embedding $\varphi_{m}: Y \hookrightarrow X$ contains already $x_{1}, \ldots, x_{m}$ but not $x_{m+1}$, one has to chose a compact $K_{m}$ containing $x_{1}, \ldots, x_{m}$ so that $x_{m+1}$ is outside the holomorphic hull of $K_{m}$. Setting $K=K_{m}$ and taking $y$ outside the convex hull we construct an automorphism $\Psi=: \Psi_{m}$ as in Proposition 2.1 and replace $\varphi_{m}$ by $\varphi_{m+1}=\Psi_{m} \circ \varphi_{m}$. Then the image of $\varphi_{m+1}$ contains already $x_{1}, \ldots, x_{m+1}$. It turns out that compacts $K_{m}$ and automorphisms $\Psi_{m}$ can be chosen so that the limit $\psi=\lim _{m \rightarrow \infty} \varphi_{m}$ is also a proper holomorphic embedding which implies the desired conclusion.

It is also worth mentioning that by a result of WINKELMANN [73] (generalizing the earlier results of RosAY and RUDIN [58] and the first author [42]), there is a discrete subset $E$ of $X$ whose complement is $n$-Eisenman hyperbolic. Hence there exists a proper embedding $\psi: Y \hookrightarrow X$ with $n$-Eisenman hyperbolic complement $X \backslash \psi(Y)$.

We continue now the list of further applications of Theorem 2 in the case where $X$ is Euclidean space $\mathbb{C}^{n}, n>1$.

(1) Property (E) yields a counterexample to the analytic version of the AbhyankarMoh-Suzuki theorem which states that every polynomial embedding of $\mathbb{C}$ into $\mathbb{C}^{2}$ is rectifiable, i.e. the image can be sent to a coordinate line by a polynomial automorphism of $\mathbb{C}^{2}$. However, there exists a proper non-rectifiable holomorphic embedding $\psi: \mathbb{C} \hookrightarrow \mathbb{C}^{2}\left[34\right.$. Indeed, according to $(\mathrm{E})$ we can make $\mathbb{C}^{2} \backslash \psi(\mathbb{C})$ 2-Eisenman hyperbolic while the complement $\mathbb{C}^{*} \times \mathbb{C}^{2}$ to the coordinate line is not. In fact, the complement $\mathbb{C}^{2} \backslash \psi(\mathbb{C})$ can be made Kobayashi hyperbolic.

Furthermore, there exist uncountably many non-rectifiable embeddings in a reasonable sense. Let us be more precise.

2.6. Definition. Two proper holomorphic embeddings $\Phi, \Psi: X \hookrightarrow \mathbb{C}^{n}$ are equivalent if there exist holomorphic automorphisms $\varphi \in \operatorname{Authol}_{\text {hol }}\left(\mathbb{C}^{n}\right)$ and $\psi \in \operatorname{Aut}_{\text {hol }}(X)$ such that $\varphi \circ \Phi=\Psi \circ \psi$.

2.7. Remark. We would like to emphasize that there is another (weaker) definition of equivalence. It is so-called $\operatorname{Aut}\left(\mathbb{C}^{n}\right)$-equivalence which was used by several authors (e.g., Buzzard, Forstnerič, Globevnik and Varolin) who proved uncountability of certain equivalence classes of embeddings in this weaker sense. In our definition the map $\Psi^{-1} \circ \varphi \circ \Phi$ is well-defined and it is an automorphism of $X$ while for the weaker notion one has to demand that $\Psi^{-1} \circ \varphi \circ \Phi$ is the identity on $X$. 
The best known results are the following.

(2) In [8] BorELL and the second author showed that if (i) $X$ is a Stein space such that its group of holomorphic automorphisms is a Lie group (with possibly countably many components) and (ii) there exists a proper holomorphic embedding of $X$ into $\mathbb{C}^{m}$ where $0<\operatorname{dim} X=n<m$, then for any $k \geq 0$ there are uncountably many non-equivalent proper holomorphic embeddings $\Psi: X \times \mathbb{C}^{k} \hookrightarrow \mathbb{C}^{m} \times \mathbb{C}^{k}$.

(3) These non-equivalent embeddings appear even in holomorphic families. Under the same assumptions on $X$ as in (2) with $k=m-n-1$ there exists a family of holomorphic embeddings of $X \times \mathbb{C}^{l}$ into $\mathbb{C}^{m} \times \mathbb{C}^{l}$ parameterized by $\mathbb{C}^{k}$, such that for different parameters $w_{1} \neq w_{2} \in \mathbb{C}^{k}$ the embeddings $\psi_{w_{1}}, \psi_{w_{2}}: X \times \mathbb{C}^{l} \hookrightarrow \mathbb{C}^{n+l}$ are non-equivalent. This result is due to LoDIN and the second author [51] (an important ingredient of their proof is a parametric version of Theorem 2 from [49]).

It is worth mentioning that the last two results include embeddings of $\mathbb{C}^{n}$ into $\mathbb{C}^{m}$ for any $n<m$ (respectively $n<m-1$ for the families), by choosing $X=\mathbb{C}^{n}$. Thus the holomorphic analogue of the ABHYANKAR and SATHAYE problem has a negative answer.

An important application of the non-rectifiable embeddings is the construction of non-linearizable holomorphic actions of reductive Lie groups on affine spaces by DERKSEN and the second author [17], [18].

2.8. Definition. A holomorphic action of a reductive group $G$ on $\mathbb{C}^{n}$ is said to be linearizable if there exists a holomorphic automorphism $\alpha \in \operatorname{Aut}_{\text {hol }}\left(\mathbb{C}^{n}\right)$, such that $\alpha \circ g \circ \alpha^{-1} \in G L_{n}(\mathbb{C})$ for every $g \in G$.

(4) For any nontrivial complex reductive Lie group $G$ there is a natural $N$ such that for all $n \geq N$ there is a non-linearizable holomorphic $G$-action on $\mathbb{C}^{n}$. The optimal dimension (minimal $N$ ) is not known for any $G$ including $G=\mathbb{C}^{*}$. All holomorphic actions on $\mathbb{C}^{2}$ are linearizable by a result of SUzUKI [65] but starting with dimension $n=4$ there are non-linearizable actions on $\mathbb{C}^{n}$. The problem of linearization of holomorphic $\mathbb{C}^{*}$-actions on $\mathbb{C}^{3}$ is still open while all algebraic $\mathbb{C}^{*}$-actions on $\mathbb{C}^{3}$ are known to be linearizable 43 .

\subsection{Sketch of a construction of a non-linearizable holomorphic $\mathbb{C}^{*}$-action on}

$\mathbb{C}^{4}$. Suppose $\varphi: \mathbb{C} \rightarrow \mathbb{C}^{2}$ is a proper holomorphic embedding. Consider a pseudoaffine modification $X$ of $\mathbb{C}_{x, y, u}^{3}$ along the divisor $D:=\{u=0\}=\mathbb{C}^{2} \times\{0\}$ with center $\varphi(\mathbb{C}) \subset D$. That is, if $f \in \operatorname{Hol}(D)$ is a holomorphic function generating the principal ideal of functions vanishing on $\varphi(\mathbb{C}) \subset D$, then $X$ is biholomorphic to the submanifold of $\mathbb{C}_{x, y, u, v}^{4}$ given by the equation $f(x, y)=u v$. One of crucial facts observed 
by Asanuma is that $X \times \mathbb{C}$ is biholomorphic to $\mathbb{C}^{4}[5$, because $X \times \mathbb{C}$ is the pseudoaffine modification of $\mathbb{C}^{4}$ along the divisor $\mathbb{C}^{3} \times\{0\}$ with center $\varphi(\mathbb{C}) \times\{0\} \subset \mathbb{C}^{3}$ and any proper holomorphic embedding of $\mathbb{C}$ into $\mathbb{C}^{n}$ with the image contained in a hyperplane is rectifiable (see also [47]). Consider the $\mathbb{C}_{\lambda}^{*}$-action on $X \times \mathbb{C}_{w}$ given by $\lambda(x, y, u, v, w)=\left(x, y, \lambda^{2} u, \lambda^{-2} v, \lambda w\right)$. The categorical quotient $\mathbb{C}^{4} / / \mathbb{C}_{\lambda}^{*}$ of this action is equipped with a natural so-called Luna stratification for which one of the strata isomorphic to $\mathbb{C}$ is contained in a higher-dimensional stratum isomorphic to $\mathbb{C}^{2}$ exactly in the same manner as $\mathbb{C} \simeq \varphi(\mathbb{C})$ is contained in $D \simeq \mathbb{C}^{2}$. In the case of a linearizable action this first stratum must be rectifiable in the second one. However by (1) we can suppose that $\varphi(\mathbb{C})$ is not rectifiable in $D$ which yields a non-linearizable holomorphic $\mathbb{C}^{*}$-action.

2.10. Remark. The linearization problem for $\mathbb{C}^{*}$-actions on $\mathbb{C}^{3}$ is related to the question whether the pseudo-affine modification $X$ of $\mathbb{C}^{3}$ as before is biholomorphic to $\mathbb{C}^{3}$. If the answer is positive we had a non-linearizable action on $\mathbb{C}^{3}$, otherwise it is a counterexample to the holomorphic analogue of Zariski's cancellation problem (i.e. the question whether a complex manifold $Y$ is biholomorphic to $\mathbb{C}^{n}$ provided that $Y \times \mathbb{C}^{k}$ is biholomorphic to $\left.\mathbb{C}^{k+n}\right)$. Returning to $X$ we note that it is diffeomorphic to $\mathbb{R}^{6}$ as a smooth real manifold ([44, Appendix]) and it has the density property by Theorem 3 , By a conjecture of VAROLIN and ToTH [67] such an $X$ must be biholomorphic to $\mathbb{C}^{3}$, i.e. in the case of a negative answer we can disprove their hypothesis. More potential counterexamples to the conjecture of VAROLIN and ToTH can be found in [44]. One of the most interesting among them is a modification whose center is the RUSSELL cubic (more precisely, this modification is isomorphic to the algebraic hypersurface in $\mathbb{C}^{6}$ given by the equation $\left.x+x^{2} y+s^{2}+t^{3}=u v\right)$. It is again diffeomorphic to $\mathbb{R}^{10}$ and has the density property but it is even unknown whether it is different from $\mathbb{C}^{5}$ as an algebraic variety.

(5) Similar reasoning as before leads from families of holomorphic embeddings like in (3) to families of pairwise non-equivalent $\mathbb{C}^{*}$-actions. For example, there is a family $\mathbb{C}_{w} \times \mathbb{C}^{*} \rightarrow \operatorname{Aut}_{\text {hol }}\left(\mathbb{C}^{5}\right)$ of holomorphic $\mathbb{C}^{*}$-actions on $\mathbb{C}^{5}$ parametrized by $w \in \mathbb{C}$ such that for different parameters the actions are non-equivalent (i.e. they are not conjugated by an automorphism). Moreover there is a family such that the $w=0$ represents a linear action. It follows also from (2) that on $\mathbb{C}^{4}$ there are uncountably many non-equivalent $\mathbb{C}^{*}$-actions.

(6) One of the questions coming from complex dynamical systems is description of the boundaries of Fatou-Bieberbach domains. Say, a surprising result of StEnsönes 64]) provides such a domain in $\mathbb{C}^{2}$ with a smooth boundary which has, therefore, Hausdorff dimension $d=3$. Furthermore, it was established by methods of complex dynamical systems that such a dimension can take any value $3 \leq d<4$. However the question about a Fatou-Bieberbach domain in $\mathbb{C}^{2}$ with a boundary of Hausdorff dimension $d=4$ remained open until Peters and Fornæss-Wold [55] managed to 
construct it using the ANDERSÉn-LEMPERT theory.

(7) All Fatou-Bieberbach domains arising as basins of attraction (as indicated in Remark 2.5) or more generally as domains of convergence of sequences of automorphisms of $\mathbb{C}^{n}$ are always Runge domains. Thus it is natural to ask whether all Fatou-Bieberbach domains in $\mathbb{C}^{n}$ have to be Runge. This problem was solved by FornÆss-WolD who constructed a Fatou-Bieberbach domain in $\mathbb{C} \times \mathbb{C}^{*}$ which is not Runge in $\mathbb{C}^{2}$ (but Runge in $\left.\mathbb{C} \times \mathbb{C}^{*}\right)$ using the density property of $\mathbb{C} \times \mathbb{C}^{*}[74$.

(8) Developing the ideas from (7) further FORNÆSS-WOLD constructed also a "long $\mathbb{C}^{2}$ " which is not biholomorphic to $\mathbb{C}^{2}$, thus solving a classical open question. By a "long $\mathbb{C}^{2 "}$ we mean a complex manifold $X$ which can be exhausted by open subsets $\Omega_{i}$ which are all biholomorphic to $\mathbb{C}^{2}$, i.e. $X=\bigcup_{i=1}^{\infty} \Omega_{i}, \Omega_{i} \subset \Omega_{i+1}$, and $\Omega_{i} \cong \mathbb{C}^{2}$ for all $i \in \mathbb{N}$. Here of course $\Omega_{i} \subset \Omega_{i+1}$ is not a Runge pair.

(9) A beautiful combination of differential-topological methods with hard analysis (solutions of $\bar{\partial}$-equations with exact estimates) and the Andersén-Lempert-theory is required for understanding of how many totally real differentiable embeddings of a real manifold $M$ into $\mathbb{C}^{n}$ can exist.

If $f_{0}, f_{1}: M \rightarrow \mathbf{C}^{n}$ are two totally real, polynomially convex real-analytic embeddings of a compact manifold $M$ into $\mathbb{C}^{n}$, we say that $f_{0}$ and $f_{1}$ are Aut ${ }_{\text {hol }}\left(\mathbb{C}^{n}\right)$-equivalent if $f_{1}=F \circ f_{0}$, where $F: U \rightarrow F(U) \subset \mathbf{C}^{n}$ is a biholomorphism defined in a neighbourhood $U$ of $f_{0}(M)$ such that $F$ is the uniform limit in $U$ of a sequence of elements of $\operatorname{Aut}_{\text {hol }}\left(\mathbb{C}^{n}\right)$. Conditions for Aut $\mathrm{Aol}\left(\mathbb{C}^{n}\right)$-equivalence were found in [29], using volume-preserving automorphisms (and an approach using automorphisms preserving the holomorphic symplectic form was considered in [31]).

In the smooth case let $\mathcal{E}^{r}\left(M, \mathbf{C}^{n}\right)$ be the set of all totally real polynomially convex $C^{r}$-embeddings of $M$ into $\mathbb{C}^{n}$ (for $2 \leq r \leq \infty$ ). It is proved by Forstnerič and LÖW that two embeddings $f_{0}, f_{1} \in \mathcal{E}^{\infty}\left(M, \mathbb{C}^{n}\right)$ belong to the same connected component (in the space of $C^{r}$-embeddings of $M$ into $\mathbb{C}^{n}$ equipped with the usual topology of uniform convergence of all derivative up to order $r$ ) if and only if there exists a sequence $\left\{\Phi_{j}\right\} \subset$ Aut $_{\text {hol }}\left(\mathbb{C}^{n}\right)$ such that $\Phi_{j} \circ f_{0} \rightarrow f_{1}$ and $\Phi_{j}^{-1} \circ f_{1} \rightarrow f_{0}$ in $C^{\infty}(M)$ as $j \rightarrow \infty$. Precise results in the case $r<\infty$ were obtained in [33].

(10) Another interesting problem is to find embeddings of Stein manifolds into $\mathbb{C}^{m}$ with prescribed interpolation condition on discrete subsets. This is much more difficult than just letting the image contain a given discrete subset (not caring about the preimage points). Beside Theorem 2 some properties of $\mathbb{C}^{m}$ were used by ForstnerIČ,

\footnotetext{
${ }^{4}$ It is unfortunate that in the literature the term "Authol $\left(\mathbb{C}^{n}\right)$-equivalence" is used in different meanings - another one was mentioned in Remark 2.7.
} 
IVARsson, Prezelu and the second author in 28] to prove the result below (for general targets with the density property the problem is still widely open).

Let $X$ be a Stein manifold of dimension $n>1,\left\{a_{j}\right\}_{j \in \mathbb{N}}\left(\right.$ resp. $\left.\left\{b_{j}\right\}_{j \in \mathbb{N}}\right)$ be a sequence of distinct points in $X$ (resp. $\left.\mathbb{C}^{m}\right)$. If $m \geq N=\left[\frac{3 n}{2}\right]+15$ then there exists a proper holomorphic embedding $f: X \hookrightarrow \mathbb{C}^{m}$ satisfying

$$
f\left(a_{j}\right)=b_{j} \text { for } j=1,2, \ldots
$$

A more general result is that if $X$ admits a proper holomorphic embedding into $\mathbb{C}^{m}$ and $\left\{a_{j}\right\}_{j \in \mathbb{N}}$ and $\left\{b_{j}\right\}_{j \in \mathbb{N}}$ are sequences as before such that $\left\{b_{j}\right\}_{j \in \mathbb{N}}$ form a so-called tame subset in $\mathbb{C}^{m}$ (by definition there is a holomorphic automorphism of $\mathbb{C}^{m}$ mapping this sequence onto the set of the integer points in a coordinate axis), then the conclusion remains true. Other results in this direction can be found in [50].

(11) A question posed by SIU asks whether there exists always a Fatou-Bieberbach domain contained in the complement to a closed algebraic subvariety $Z$ of $\mathbb{C}^{n}$ such that $\operatorname{dim} Z \leq n-2$. The affirmative answer was obtained by BUzzARD and HuBBARD who used some concrete construction. Another proof of this fact was given by the authors who used a version of the density property for such complements (see Theorem 9 below). More precisely for any point $x \in \mathbb{C}^{n} \backslash Z$ there is a Fatou-Bieberbach (i.e. holomorphic injective) map $f: \mathbb{C}^{n} \rightarrow \mathbb{C}^{n} \backslash Z$ with $f(0)=x$ (actually, the crucial fact that guarantees such maps is existence of sprays on $\mathbb{C}^{m} \backslash Z$ which can be extracted from the earlier papers of Gromov [37] and Winkelmann [72]).

In particular all Eisenman measures on $\mathbb{C}^{n} \backslash Z$ are trivial. It is worth mentioning that closed analytic subsets of $\mathbb{C}^{n}$ of codimension $k$ may have $k$-Eisenman hyperbolic complements. More precisely, it was shown in [8] that if a complex manifold $Y$ admits a proper holomorphic embedding into $\mathbb{C}^{n}$ then it has also another proper holomorphic embedding with $(n-\operatorname{dim} Y)$-Eisenman hyperbolic complement to the image (the proof is based on the Andersén-Lempert theory and a generalized idea from [16] ).

(12) The classical approximation theorem of CARLEMAN states that for each continuous function $\lambda: \mathbb{R} \rightarrow \mathbb{C}$ and a positive continuous function $\varepsilon: \mathbb{R} \rightarrow(0, \infty)$ there exists an entire function $f$ on $\mathbb{C}$ such that $|f(t)-\lambda(t)|<\varepsilon(t)$ for every $t \in \mathbb{R}$.

Using the Andersén-Lempert-theory together with some explicit shears (which are special automorphisms of $\mathbb{C}^{n}$ which appear in that theory) BuzzARD and Forstnerič [15] were able to prove a similar result for holomorphic automorphisms of $\mathbb{C}^{n}$. Namely, for any proper embedding $\lambda: \mathbb{R} \rightarrow \mathbb{C}^{n}$ of class $C^{r}$ (where $n \geq 2$ and $r \geq 0$ ) and a positive continuous function $\varepsilon: \mathbb{R} \rightarrow(0, \infty)$ there exists a proper holomorphic embedding $f: \mathbb{C} \rightarrow \mathbb{C}^{n}$ such that

$$
\left|f^{(s)}(t)-\lambda^{(s)}(t)\right|<\varepsilon(t) \quad \forall t \in \mathbb{R}, 0 \leq s \leq r .
$$

\footnotetext{
${ }^{5}$ Such $N$ is chosen because it is the optimal embedding dimension, see (14) below.
} 
Actually this fact remains valid under the additional requirement that the embedding satisfies the interpolation property as in (10).

(13) If $Z$ is a smooth closed algebraic subvariety of $\mathbb{C}^{n}$ such that $n>2 \operatorname{dim} Z+1$ then it is known [41] (see also [63]) that any automorphism of $Z$ extends to an automorphism of $\mathbb{C}^{n}$. In the holomorphic category the situation is completely different and exploiting Theorem 2 the second author showed in [19] that there is a proper holomorphic embedding of $\varphi: \mathbb{C} \hookrightarrow \mathbb{C}^{n}$ with any $n \geq 2$ (thus the codimension is arbitrarily big) such that the only automorphism of $\mathbb{C}^{n}$ mapping the image onto itself is the identical one.

(14) The classical theorem of REMMERT [56] states that any Stein manifold $Y$ of dimension $n$ admits a proper holomorphic embedding into a Euclidean space $\mathbb{C}^{N}$ of sufficiently high dimension $N$. The optimal $N$ was found in the papers of Gromov, EliashberG [25] and SCHÜRMANN [60]; in the case of $n \geq 2$ they proved that $Y$ can be embedded into $\mathbb{C}^{[3 n / 2]+1}$. This result is sharp by virtue of examples of O. Forster [27], who conjectured that the optimal $N$ was $[3 n / 2]+1$. The case of $n=1$ is still open, i.e. it is unknown whether any open Riemann surface admits a proper holomorphic embedding into $\mathbb{C}^{2}$. However, there was a recent breakthrough in this direction FORNÆSS WOLD proved the Forster conjecture for all finitely connected domains in $\mathbb{C}$ and for all elliptic curves with finitely many holes (that are not punctures) using the Andersén-Lempert theory in a very clever way [75], [76], [77]. Furthermore, Löw, FORNAESS-WOLD and the second author [52] showed that one can require additionally an interpolation condition on discrete subsets as in (10).

\section{Criterion For the Algebraic Density PROPERTy.}

Tотн and VAROLIN established the algebraic density property for some manifolds including semi-simple complex Lie groups [67], 68]. Their proof follows to a great extend the original ideas of ANDERSÉn and LEMPERT and is quite complicated. A new approach suggested by the authors [45] lead to the following.

Theorem 4. Each linear algebraic group whose connected component is different from $\mathbb{C}_{+}$or $\left(\mathbb{C}^{*}\right)^{k}, k \geq 1$ has the algebraic density property.

The proof was based on the following simple fact.

Theorem 5. Let $X$ be an affine algebraic manifold with a transitive group Aut $X$ of algebraic automorphisms and let $\mathbb{C}[X]$ be its algebra of regular functions. Suppose that there is a submodule $L$ of the $\mathbb{C}[X]$-module $T$ of all algebraic vector fields on $X$ such that $L \subset \operatorname{Lie}_{\text {alg }}(X)$ and the fiber of $L$ at some point $x_{0} \in X$ contains a generating subse 6 of $T_{x_{0}} X$. Then $X$ has the algebraic density property.

\footnotetext{
${ }^{6}$ Our notion of a generating subset is milder than usual. A finite subset $F$ of $T_{x_{0}} X$ is called a generating subset if the span of the orbit of $F$ under the action of the isotropy group $(\text { Aut } X)_{x_{0}}$ coincides with $T_{x_{0}} X$ (say, if $X$ is a simple Lie group then every nonzero vector is a generating set.) .
} 
Proof. The action of $\alpha \in$ Aut $X$ maps $L$ onto another $\mathbb{C}[X]$-module $L_{\alpha}$. The sum of such modules with $\alpha$ running over a subset of Aut $X$ is again a $\mathbb{C}[X]$-submodule $N$ of $T$. Let $\mathcal{M}_{x} \subset \mathbb{C}[X]$ be the maximal ideal that consists of functions vanishing at $x \in X$. By assumption $N$ can be chosen so that $N / \mathcal{M}_{x_{0}} T$ coincides with $T_{x_{0}} X=T / \mathcal{M}_{x_{0}} T$. Furthermore, since $X$ is homogeneous with respect to Aut $X$ we can suppose that the same is true for every point in $X$. That is, for the $\mathbb{C}[X]$-module $Q=T / N$ and every $x \in X$ we have $Q / \mathcal{M}_{x} Q=0$. Thus $Q=0$ and $N=T$ (e.g., see [39, Exercise II.5.8]). Since composition with automorphisms preserves complete integrability, all element of $N$ are in $\operatorname{Lie}_{\text {alg }}(X)$ which implies the desired conclusion.

Thus the idea of the proof of Theorem 4 is to catch a nontrivial $\mathbb{C}[X]$-module in the $\operatorname{Lie}_{a l g}(X)$. In order to demonstrate how to do it we prove the main observation of the Andersén-Lempert theory.

3.1. Proposition. For $n \geq 2$ the space $\mathbb{C}^{n}$ has the algebraic density property.

Proof. Let $x_{1}, \ldots, x_{n}$ be a coordinate system on $X=\mathbb{C}^{n}$ and $\delta_{i}=\partial / \partial x_{i}$, i.e. $\operatorname{Ker} \delta_{i}=$ $\mathbb{C}\left[x_{1}, \ldots, \hat{x}_{i}, \ldots, x_{n}\right]$ and therefore

$$
\mathbb{C}^{[n]}=\operatorname{Span} \operatorname{Ker} \delta_{1} \cdot \operatorname{Ker} \delta_{2} .
$$

Note also that for $f_{i} \in \operatorname{Ker} \delta_{i}$ the algebraic vector fields $f_{i} \delta_{i}$ and $x_{i} f_{i} \delta_{i}$ are completely integrable on $\mathbb{C}^{n}$. This implies that the field

$$
\left[f_{1} \delta_{1}, x_{1} f_{2} \delta_{2}\right]-\left[x_{1} f_{1} \delta_{1}, f_{2} \delta_{2}\right]=f_{1} f_{2} \delta_{2}
$$

belongs to $\operatorname{Lie}_{a l g}(X)$. Thus $\operatorname{Lie}_{\text {alg }}(X)$ contains all algebraic fields proportional to $\delta_{2}$ which in combination with Theorem 5 implies the desired conclusion.

To transfer this argument to other affine algebraic manifolds we have to use locally nilpotent and semi-simple derivations instead of partial derivatives. Recall that an algebraic vector field $\sigma$ on $X$ is locally nilpotent (LND) if its flow is an algebraic $\mathbb{C}_{+}-$ action. Equivalently, $\sigma$ is a LND if for every $a \in \mathbb{C}[X]$ there exists natural $n$ for which $\sigma^{n}(a)=0$. The last algebraic definition enables us to introduce the degree of any regular element $a \in \mathbb{C}[X]$ with respect to $\sigma$ as $\operatorname{deg}_{\sigma}(a)=\min \left\{n-1 \mid \sigma^{n}(a)=0\right\}$.

An algebraic vector field on $X$ is semi-simple if its flow is an algebraic $\mathbb{C}^{*}$-action.

3.2. Definition. Let $\sigma$ be a LND on $X$ and $\delta$ be either a LND or semi-simple.

Then pair $(\sigma, \delta)$ is called semi-compatible if the the span of $\operatorname{Ker} \sigma \cdot \operatorname{Ker} \delta$ contains a nonzero ideal of $\mathbb{C}[X]$.

A semi-compatible pair $(\sigma, \delta)$ is called compatible if one of the following conditions holds:

(1) there exists $a \in \mathbb{C}[X]$ such that $a \in \operatorname{Ker} \delta$ and $\sigma(a) \in \operatorname{Ker} \sigma \backslash 0$, i.e. $\operatorname{deg}_{\sigma}(a)=1$.

(2) both $\sigma$ and $\delta$ are LNDs and there exists $a \in \mathbb{C}[X]$ such that $\operatorname{deg}_{\sigma}(a)=1=$ $\operatorname{deg}_{\delta}(a)$. 
Repeating the argument from Proposition 3.1 with $\sigma$ and $\delta$ instead of $\delta_{1}$ and $\delta_{2}$ we get the following.

3.3. Proposition. The existence of a compatible pair yields the existence of a nontrivial $\mathbb{C}[X]$-module in $\operatorname{Lie}$ alg $(X)$.

Now we can formulate our criterion as the following.

Theorem 6. Let $X$ be a smooth affine algebraic variety with a transitive automorphism group Aut $X$. Suppose that there are finitely many pairs of compatible vector fields $\left\{\sigma_{i}, \delta_{i}\right\}$ such that at some point $x_{0} \in X$ vectors $\left\{\delta_{i}\left(x_{0}\right)\right\}$ form a generating subset of $T_{x_{0}} X$. Then $X$ has the algebraic density property.

3.4. Example. (1) As an obvious application of this theorem we see that the manifold $X=\mathbb{C}^{k} \times\left(\mathbb{C}^{*}\right)^{l}$ with $k \geq 1$ and $k+l \geq 2$ has algebraic density property.

(2) A more interesting case is when $X=S L_{2}$ (or $P S L_{2}$ ). Denote by

$$
A=\left(\begin{array}{ll}
a_{1} & a_{2} \\
b_{1} & b_{2}
\end{array}\right)
$$

an element of $X$. Consider the following LNDs on $\mathbb{C}[X]$ :

$$
\begin{aligned}
\delta_{1} & =a_{1} \frac{\partial}{\partial b_{1}}+a_{2} \frac{\partial}{\partial b_{2}} \\
\delta_{2} & =b_{1} \frac{\partial}{\partial a_{1}}+b_{2} \frac{\partial}{\partial a_{2}} .
\end{aligned}
$$

Then $\operatorname{Ker} \delta_{1}=\mathbb{C}\left[a_{1}, a_{2}\right]$ and $\operatorname{Ker} \delta_{2}=\mathbb{C}\left[b_{1}, b_{2}\right]$, i.e. Span Ker $\delta_{1} \cdot \operatorname{Ker} \delta_{2}=\mathbb{C}[X]$ and the pair $\left(\delta_{1}, \delta_{2}\right)$ is semi-compatible.

Furthermore, $\operatorname{deg}_{\delta_{1}}(a)=\operatorname{deg}_{\delta_{2}}(a)=1$ for $a=a_{1} b_{2}$ and therefore the pair $\left(\delta_{1}, \delta_{2}\right)$ is compatible. Hence $S L_{2}$ and $P S L_{2}$ have the algebraic density property. It can be shown in the same manner that $S L_{n}$ and $P S L_{n}$ have also algebraic density property.

3.5. Definition. Let us choose an identification of elements of $S L_{2}$ with $(2 \times 2)$-matrices with determinant 1 . Suppose that $H_{1} \simeq \mathbb{C}_{+}$(resp. $H_{2} \simeq \mathbb{C}_{+}$) is the unipotent upper (resp. lower) triangular subgroup of $S L_{2}$. Then any $S L_{2}$-action on $X$ generates $H_{i^{-}}$ action on $X$ and, therefore, a locally nilpotent vector field $\delta_{i}$. The pair $\left(\delta_{1}, \delta_{2}\right)$ will be called an associated pair of LNDs of the $S L_{2}$-action.

Theorem 7. Let $X$ be a smooth complex affine algebraic variety whose group of algebraic automorphisms is transitive.

(1) Suppose that $X$ is equipped with a non-degenerate fixed point free $S L_{2}$-action. Then the associated pair $\left(\delta_{1}, \delta_{2}\right)$ is compatible.

(2) Suppose that $X$ is equipped with $N$ non-degenerate fixed point free $S L_{2}$-actions. Let $\left\{\delta_{1}^{k}, \delta_{2}^{k}\right\}_{k=1}^{N}$ be the corresponding pairs of associated locally nilpotent vector fields.

If $\left\{\delta_{2}^{k}\left(x_{0}\right)\right\}_{k=1}^{N} \subset T_{x_{0}} X$ is a generating set at some point $x_{0} \in X$ then $X$ has the algebraic density property.

\footnotetext{
${ }^{7}$ That is, the dimension of general orbits is 3 .
} 
The second statement is, of course, a consequence of (1) and Theorem 6. The idea of the proof of the first statement can be described as follows. By LunA's slice theorem (e.g., see [22]) any closed $S L_{2}$-orbit $O$ possesses an $S L_{2}$-invariant neighborhood $U^{\prime}$ for which there exists a surjective étale $S L_{2}$-equivariant morphism $U^{\prime \prime} \rightarrow U^{\prime}$ such that $U^{\prime \prime}$ is naturally isomorphic to $S L_{2} \times_{I_{x}} V 8$ where $I_{x} \subset S L_{2}$ is the isotropy group of some point $x \in O$ and $V$ is an $I_{x}$-invariant subvariety of $X$ (called a slice). Then the associated locally nilpotent derivations $\delta_{1}$ and $\delta_{2}$ generate naturally similar derivations $\delta_{1}^{\prime}$ and $\delta_{2}^{\prime}$ ( resp. $\delta_{1}^{\prime \prime}$ and $\delta_{2}^{\prime \prime}$ ) on $U^{\prime}$ (resp. $U^{\prime \prime}$ ). The straightforward application of Nullstellensatz shows that $\delta_{1}$ and $\delta_{2}$ are compatible provided that for every closed orbit $O$ the pair $\left(\delta_{1}^{\prime}, \delta_{2}^{\prime}\right)$ described before is compatible. It was shown in [45] that compatibility of $\delta_{1}^{\prime}$ and $\delta_{2}^{\prime}$ follows from compatibility of $\delta_{1}^{\prime \prime}$ and $\delta_{2}^{\prime \prime}$. The proof of compatibility of the associated locally nilpotent derivations for the natural $S L_{2}$-action on $S L_{2} \times_{I_{x}} V$ (especially in the case when $I_{x}$ contains $\mathbb{C}^{*}$ ) is the most difficult part which does not work for $S L_{2}$-actions with fixed points. Actually, if a non-degenerate $S L_{2}$-action has a fixed point the pair $\left(\delta_{1}^{\prime}, \delta_{2}^{\prime}\right)$ is not compatible.

As a (non-straightforward) application of Theorem 7 we have.

Theorem 8. Let $G$ be a linear algebraic group and $R$ be its proper reductive subgroup.

Suppose that the connected components of $X=G / R$ are different from $\mathbb{C}_{+}$or $\left(\mathbb{C}^{*}\right)^{k}$.

Then $X$ has the algebraic density property.

The difficulty in the proof of the last result lies in checking the assumption of Theorem 7. That is, one needs to find an $S L_{2}$-subgroup $\Gamma$ of $G$ such that its natural action on $X$ is fixed point free and non-degenerate. This is equivalent to the next fact.

3.6. Proposition. Let $G$ and $R$ be as before. Then there exists an $S L_{2}$-subgroup $\Gamma$ of $G$ such that

(1) $g \Gamma g^{-1}$ is not contained in $R$ for any $g \in G$ and

(2) $g_{0} \Gamma g_{0}^{-1}$ meets $R$ at a finite set for some $g_{0} \in G$.

Furthermore $\Gamma$ can be chosen as a principal or sub-regular $S L_{2}$-subgroup of $G$.

The proof of Proposition 3.6 is surprisingly non-trivial. First using the JackobsonMorozov theorem (e.g., see [9]) we can switch from studying the conjugate classes of $S L_{2}$-subgroups in $G$ to the study of orbits of nilpotent elements in the Lie algebra of $G$ under the adjoint action. The orbit of the largest dimension is called principal and the second largest dimension sub-regular (they are both unique). To show that an $S L_{2}$-subgroup corresponding to one of these orbits satisfy conditions (1) and (2) one needs to use the Dynkin classification of nilpotent orbits (e.g. see [6] and [7]) in combination with some difficult facts about the Freudental square [53].

Besides Theorem 7 (1) we have another useful criterion for compatibility [45, Proposition 3.9].

\footnotetext{
${ }^{8}$ That is, elements of $U^{\prime \prime}$ are equivalence classes in $S L_{2} \times V$ given by the relation $(s, v) \sim\left(s g^{-1}, g \cdot v\right)$ for $g \in I_{x}$.
} 
3.7. Proposition. Suppose that either $\Gamma=S L_{2}$ and $H_{1}, H_{2}$ are from Definition 3.5 or $\Gamma=H_{1} \times H_{2}$ where $H_{1} \simeq \mathbb{C}_{+}$and $H_{2}$ is one of the groups $\mathbb{C}_{+}$or $\mathbb{C}^{*}$. Let $X$ be a smooth affine algebraic $\Gamma$-variety and $Y$ be a normal affine algebraic variety equipped with a trivial $\Gamma$-action. Let $r: X \rightarrow Y$ be a surjective $\Gamma$-equivariant morphism and $\delta_{1}, \delta_{2}$ be the algebraic fields on $X$ generated by the action of $H_{1}$ and $H_{2}$. Suppose that for every point $y \in Y$ there exists an étale neighborhood $W \rightarrow Y$ such that the pair of vector fields induced by $\delta_{1}$ and $\delta_{2}$ on the fibered product $X \times_{Y} W$ is compatible. Then the pair $\left(\delta_{1}, \delta_{2}\right)$ is compatible.

\subsection{Non-Stein Case: Complement to a Subvariety of Codimension at least}

2. Let $Y$ be an algebraic subvariety of an affine algebraic manifold $X$ such that $\operatorname{codim}_{X} Y \geq 2$. Then according to Forstnerič $X \backslash Y$ has the algebraic density property if the Lie algebra $\operatorname{Lie}_{\text {alg }}(X, Y)$ generated by completely integrable algebraic vector fields on $X$ that vanishes on $Y$ coincides with the Lie algebra $\operatorname{VF}_{\text {alg }}(X, Y)$ of all algebraic vector fields on $X$ that vanishes on $Y$.

3.9. Remark. By Hartogs' theorem every algebraic vector field on $X \backslash Y$ can be extended to $X$. In the case of completely integrable fields this extension must be tangent to $Y$. In particular, $\operatorname{Lie}_{\text {alg }}(X, Y)$ cannot contain algebraic fields with extension non-tangent to $Y$. Furthermore, in the case of $Y$ without the algebraic density property (say, when $Y$ is singular) $\operatorname{Lie}_{\text {alg }}(X, Y)$ cannot contain all vector fields tangent to $Y$. Thus the Forstnerič definition is the only reasonable.

Theorem 9. (45]) Let $Y$ be a subvariety of $\mathbb{C}^{n}$ of codimension $\geq 2$. Then

(1) $\mathbb{C}^{n} \backslash Y$ has the algebraic density property if $T_{y} Y \leq n-1$ for every $y \in Y$;

(2) in any case there exists natural $k$ such that $\operatorname{Lie}_{a l g}\left(\mathbb{C}^{n}, Y\right)$ contains all algebraic vector fields that vanish on $Y$ with multiplicity at least $k$.

It can be shown that even under condition (2) the approximation theorems we had in the affine case are valid for $\mathbb{C}^{n} \backslash Y$.

\section{Volume DENSITY PROPERTY}

As we mentioned before, the algebraic volume density property (see Definition 1.2) is much more delicate thing to prove than the algebraic density property. It is hopeless to look for a $\mathbb{C}[X]$-module inside the Lie algebra $\operatorname{Lie}_{\text {alg }}^{\omega}(X)$ generated by completely integrable algebraic vector fields of divergence zero with respect to an algebraic volume form $\omega$ on $X$. Indeed, let $\nu$ be a vector field on $X$ of $\omega$-divergence zero and $f \in \mathbb{C}[X]$. Then $\operatorname{div}_{\omega}(f \nu)=\nu(f)$ is nonzero for general $f$. However, some important facts remain valid in this volume-preserving case [46].

Theorem 10. Let $X$ be a smooth hypersurface in $\mathbb{C}_{u, v, \bar{x}}^{n+2}$ given by an equation uv $=p(\bar{x})$. Suppose that $Z \subset \mathbb{C}_{\bar{x}}^{n}$ is given by $p(\bar{x})=0$ and $H^{n-2}(Z, \mathbb{C})=0$. Then $X$ has the algebraic volume density property. 
Note that unlike in Theorem 3 we have an extra assumption on the zero locus $Z$; namely $H^{n-2}(Z, \mathbb{C})=0$. It is unknown whether this assumption is essential. However this is enough to show that $S L_{2}$ (given by equation $u v=x y+1 \mathrm{in} \mathbb{C}^{4}$ ) and $P S L_{2}$ have the algebraic volume density property with respect to the invariant volume form which is important in the proof of the next result [46].

Theorem 11. Every linear algebraic group has the algebraic volume density property with respect to the left-invariant (or right-invariant) volume form.

The proof uses also the second statement of the following theorem which is not at all obvious in the volume-preserving case.

Theorem 12. (1) For an affine algebraic manifold $X$ equipped with an algebraic volume form $\omega$ the algebraic volume density property implies the volume density property (in the holomorphic sense).

(2) If affine algebraic manifolds $X$ and $Y$ have the algebraic volume density property with respect to volume forms $\omega_{X}$ and $\omega_{Y}$, then so does $X \times Y$ with respect to the volume form $\omega_{X} \times \omega_{Y}$.

Sketch of the Proof of (1). Let $\mu$ be a holomorphic vector field such that $\mu(\omega)=0$. Our aim is to find an algebraic vector field $\nu$ with $\nu(\omega)=0$ that approximates $\mu$.

In the first step one can show that there is a natural duality between the holomorphic (resp. algebraic) vector fields of $\omega$-divergence zero and the closed $(n-1)$-forms on $X$ given by $\mu \rightarrow \iota_{\mu}(\omega)$ where $\iota_{\mu}$ is the inner product (see [46, Lemmas 3.5]).

Next one needs to use the remarkable GrothendiECK's theorem [38] that states that the computation of de Rham cohomology on a smooth affine algebraic variety can be achieved via the complex of algebraic forms. Thus there exists an algebraic $(n-1)$ form $\tau_{n-1}$ such that $\iota_{\mu}(\omega)-\tau$ is exact and equal to $\mathrm{d} \tau_{n-2}$ where $\tau_{n-2}$ is a holomorphic $(n-2)$-form. Approximate $\tau_{n-2}$ by an algebraic form $\tau_{n-2}^{\prime}$ and set $\nu$ equal to the dual of the form $\tau_{n-1}+\mathrm{d} \tau_{n-2}^{\prime}$.

Instead of proving (2) we present a more complicated proof of its analogue in the holomorphic case mentioned in the introduction.

Theorem 13. If Stein manifolds $X$ and $Y$ have the volume density property with respect to forms $\omega_{X}$ and $\omega_{Y}$, so does $X \times Y$ with respect to the form $\omega_{X} \times \omega_{Y}$.

In preparation for the proof of Theorem 13 we must establish a number of facts. Recall that for a complex manifold $X$ and a holomorphic volume form $\omega$ on it the space of holomorphic vectors fields of $\omega$-divergence zero is denoted by $\mathrm{VF}_{\text {hol }}^{\omega}(X)$ and the Lie algebra generated by the set $\operatorname{IVF}_{\text {hol }}^{\omega}(X)$ of completely integrable holomorphic vector fields from $\mathrm{VF}_{\text {hol }}^{\omega}(X)$ is denoted by $\mathrm{Lie}_{\text {hol }}^{\omega}(X)$. First we shall prove the holomorphic analogue of Lemma 4.2 from [46].

4.1. Lemma. Let $\omega$ be a holomorphic form on a Stein manifold $X$ such that $X$ has the volume density property with respect to $\omega$. Then there exist finitely many vector fields 
$\delta_{1}, \delta_{2}, \ldots, \delta_{N} \in \operatorname{IVF}_{\text {hol }}^{\omega}(X)$ such that $\operatorname{Span}\left\{\delta_{i}(x) \mid i=1, \ldots, N\right\}=T_{x} X$ for every point $x \in X$.

Proof. We start with the following.

Claim. The set $\mathrm{VF}_{\text {hol }}^{\omega}(X)$ generate $T_{x} X$ for every $x \in X$.

Lets us assume first that the dimension of $X$ is bigger than one. Let $x \in X$ and $U$ be a Runge neighborhood of $x$ such that $H^{n-1}(U, \mathbb{C})=0$ where $n=\operatorname{dim} X$ (take, for instance, a small sublevel set $U$ of a strictly plurisubharmonic exhaustion function on $X$ with minimum at $x$ ). Shrinking $U$ we can assume that in some holomorphic coordinate system $z_{1}, \ldots, z_{n}$ on $U$ the form $\left.\omega\right|_{U}$ is the standard volume $\mathrm{d} z_{1} \wedge \ldots \wedge \mathrm{d} z_{n}$. Thus the holomorphic vector fields $\partial / \partial z_{i}$ on $U$ are of divergence zero and they span the tangent space at $x$. We need to approximate them by global holomorphic fields of divergence zero on $X$ which would yield our claim. As in Theorem 12 consider the inner product $\iota_{\nu}(\omega)=$ : $\alpha$ for $\nu \in \operatorname{VF}_{\text {hol }}^{\omega}(U)$. By [46, Lemma 3.5 (1)] $\alpha$ is a closed $(n-1)$-form on $U$ and since $H^{n-1}(U, \mathbb{C})=0$ we can find an $(n-2)$-form $\beta$ on $U$ with $\mathrm{d} \beta=\alpha$. Since $U$ is Runge in $X$ we can also approximate $\beta$ by a global holomorphic $(n-2)$-form $\tilde{\beta}$ (uniformly on compacts in $U$ ). Then the closed holomorphic $(n-1)$-form $\mathrm{d} \tilde{\beta}$ approximates $\alpha$ and the unique holomorphic vector field $\theta$ defined by $\iota_{\theta}(\omega)=\mathrm{d} \tilde{\beta}$ approximates $\nu$. Since $\mathrm{d} \tilde{\beta}$ is closed, the field $\theta$ is of divergence zero which concludes the proof of the Claim in the case when the dimenson of $X$ is bigger than one.

Now assume the dimension of $X$ is equal to one, i.e., $X$ is a smooth Stein curve with the volume density property. In particular, it admits a non-trivial completely integrable holomorphic vector field and, therefore, a non-constant holomorphic map $\mathbb{C} \rightarrow X$. This implies that $X$ is isomorphic to either $\mathbb{C}_{+}$or $\mathbb{C}^{*}$. In each of these cases the volume form $\omega$ for which $X$ has the volume density property is the unique (up to a constant factor) invariant volume form on the corresponding group and there exists a nowhere vanishing completely integrable vector field of $\omega$-divergence zero which concludes the proof of the Claim.

It follows from the Claim and the volume density property that vector fields from $\operatorname{Lie}_{\text {hol }}^{\omega}(X)$ span the tangent space $T_{x} X$ at any given point $x \in X$. Observe that every Lie bracket $[\nu, \mu]$ of completely integrable holomorphic vector fields of divergence zero can be approximated by a linear combination of such fields which follows immediately from the equality $[\nu, \mu]=\lim _{t \rightarrow 0} \frac{\varphi_{t}^{*}(\nu)-\nu}{t}$ where $\varphi_{t}$ is the flow generated by $\mu$. Thus the set $\operatorname{IVF}_{\text {hol }}^{\omega}(X)$ generates $T_{x} X$ at any $x \in X$.

To prove that there are finitely many fields from $\operatorname{IVF}_{\text {hol }}^{\omega}(X)$ that span each tangent space let us start with $n$ fields $\theta_{1}, \ldots, \theta_{n}$ which span the tangent space at some point $x_{0}$ and thus outside a proper analytic subset $A$. The set $A$ may have countably many irreducible components $A_{1}, A_{2}, A_{3}, \ldots$

It suffices now to find a volume preserving holomorphic automorphism $\Phi \in \operatorname{Aut}_{\text {hol }}^{\omega}(X)$ such that $\Phi(X \backslash A) \cap A_{i} \neq \varnothing$ for every $i=1,2,3, \ldots$. Indeed, for such an automorphism $\Phi$ the completely integrable holomorphic vector fields $\Phi_{*}\left(\theta_{1}\right), \ldots, \Phi_{*}\left(\theta_{n}\right)$ have divergence zero and span the tangent space at a general point in each $A_{i}$, i.e. together 
with the fields $\theta_{1}, \ldots, \theta_{n}$ they span the tangent space at each point outside an analytic subset $B$ of a smaller dimension than $A$. Then the induction by dimension implies the desired conclusion.

In order to construct $\Phi$ consider a monotonically increasing sequence of compacts $K_{1} \subset K_{2} \subset \ldots$ in $X$ such that $\bigcup_{i} K_{i}=X$ and a closed imbedding $\iota: X \hookrightarrow \mathbb{C}^{m}$. For every continuous map $\varphi: X \rightarrow \mathbb{C}^{m}$ denote by $\|\varphi\|_{i}$ the standard norm of the restriction of $\varphi$ to $K_{i}$. Let $d$ be the metric on the space $\operatorname{Aut}_{\text {hol }}(X)$ of holomorphic automorphisms of $X$ given by the formula

$$
d(\Phi, \Psi)=\sum_{i=1}^{\infty} 2^{-i}\left(\min \left(\|\Phi-\Psi\|_{i}, 1\right)+\min \left(\left\|\Phi^{-1}-\Psi^{-1}\right\|_{i}, 1\right)\right.
$$

where automorphisms $\Phi^{ \pm 1}, \Psi^{ \pm 1} \in \operatorname{Aut}_{\text {hol }}(X)$ are viewed as continuous maps from $X$ to $\mathbb{C}^{m}$. This metric makes $\operatorname{Authol}_{\text {hol }}(X)$ a complete metric space. Its subset $\operatorname{Aut}_{\text {hol }}^{\omega}(X)$ of volume-preserving automorphisms is closed and, therefore, it is a complete metric space as well.

Set $Z_{i}=\left\{\Psi \in \operatorname{Aut}_{\text {hol }}^{\omega}(X): \Psi\left(A_{i}\right) \cap(X \backslash A) \neq \varnothing\right\}$. Note that $Z_{i}$ is open in $\operatorname{Aut}_{\text {hol }}^{\omega}(X)$ and let us show that it is also everywhere dense. Indeed, the flow of any $\theta \in \operatorname{IVF}_{\text {hol }}^{\omega}(X)$ preserves $\omega$ because for any vector fields $\nu$ its divergence with respect to $\omega$ is defined by the formula $\operatorname{div}_{\omega}(\nu) \omega=L_{\nu}(\omega)$ where $L_{\nu}$ is the Lie derivative. Since $\operatorname{IVF}_{\text {hol }}^{\omega}(X)$ generates the tangent space at each point of $X$ we can choose $\theta$ non-tangent to $A_{i}$. Then for every $\Psi \in \operatorname{Aut}_{\text {hol }}^{\omega}(X)$ its composition with general elements of the flow induced by $\theta$ is in $Z_{i}$. That is, a perturbation of $\Psi$ belongs to $Z_{i}$ which proves that $Z_{i}$ is everywhere dense in $\operatorname{Aut}_{\text {hol }}^{\omega}(X)$. By the Baire category theorem the set $\bigcap_{i=1}^{\infty} Z_{i}$ is not empty which yields the existence of the desired automorphism.

4.2. Lemma. For a holomorphic field $\nu$ on $X$ denote by $\omega_{\nu}$ the inner product $\iota_{\nu}(\omega)$ of $\nu$ with the form $\omega$. If $\nu$ is of $\omega$-divergence zero then for every holomorphic function $f$ on $X$ we have $\mathrm{d}\left(\omega_{f \nu}\right)=\nu(f) \omega$.

Proof. Using the well-known formula $L_{\nu}=\mathrm{d} \circ \iota_{\nu}+\iota_{\nu} \circ \mathrm{d}$ (e.g., see [48, Proposition 3.10]) that relates the outer differentiation $\mathrm{d}$ with the Lie derivative $L_{\nu}$ and inner product $\iota_{\nu}$ we get

$$
\mathrm{d}\left(\omega_{f \nu}\right)=L_{f \nu}(\omega)-\iota_{f \nu} \mathrm{d}(\omega)=L_{f \nu}(\omega)=\operatorname{div}_{\omega}(f \nu) \omega=\left(f \operatorname{div}_{\omega}(\nu)+\nu(f)\right) \omega=\nu(f) \omega .
$$

4.3. Notation. For a complex manifold $X$ with a volume form $\omega$ we define the following linear subspace $F_{X}$ in the space of holomorphic functions $\operatorname{Hol}(X)=H^{0}(X, \mathcal{O}(X))$ on $X$ :

$$
F_{X}=\operatorname{Span}\left\{\theta(\operatorname{Hol}(X)): \theta \in \mathrm{VF}_{\text {hol }}^{\omega}(X)\right\}
$$


4.4. Lemma. Let $\Omega^{i}$ be the sheaf of holomorphic $i$-forms on a complex manifold $X$ and let $\mathcal{B}^{i} \subset \Omega^{i}$ be the subsheaf of exact $i$-forms. For $n=\operatorname{dim} X$ consider the isomorphism $\varepsilon: \operatorname{Hol}(X) \rightarrow H^{0}\left(X, \Omega^{n}\right)$ into the space $H^{0}\left(X, \Omega^{n}\right)$ of holomorphic $n$-forms given by $\varepsilon(f)=f \omega$ and suppose that

(i) the $\operatorname{Hol}(X)$-module $\mathrm{VF}_{\text {hol }}(X)$ of holomorphic vector fields on $X$ is generated by $\operatorname{VF}_{\text {hol }}^{\omega}(X)$.

Then $F_{X}=\varepsilon^{-1}\left(H^{0}\left(X, \mathcal{B}^{n}\right)\right)$ where $H^{0}\left(X, \mathcal{B}^{n}\right)$ is the space of exact $n$-forms.

Moreover, suppose that $X$ is Stein and instead of condition (i) we have

(ii) finitely many vector fields $\delta_{1}, \delta_{2}, \ldots, \delta_{N} \in \mathrm{VF}_{\text {hol }}^{\omega}(X)$ generating $T_{x} X$ at each $x \in X$.

Then $F_{X}=\operatorname{Span}\left\{\delta_{i}(\operatorname{Hol}(X)) \mid i=1, \ldots, N\right\}$.

Proof. Since $\omega$ is nowhere vanishing we have an isomorphism between the spaces of holomorphic vector fields and holomorphic $(n-1)$-forms given by $\nu \rightarrow \iota_{\nu}(\omega)$, i.e. any $(n-1)$-form can be presented as $\omega_{\nu}$ for some $\nu \in \mathrm{VF}_{\text {hol }}(X)$. Furthermore, condition (i) implies that the vector space $H^{0}\left(X, \Omega^{n-1}\right)$ is generated by elements of type $\omega_{f \delta}$ where $\delta \in \mathrm{VF}_{\text {hol }}^{\omega}(X)$ and $f \in \operatorname{Hol}(X)$. By Lemma 4.2 we have

$$
F_{X}=\operatorname{Span}\left\{\varepsilon^{-1}\left(\mathrm{~d}\left(\omega_{f \delta}\right)\right): f \in \operatorname{Hol}(X), \delta \in \mathrm{VF}_{\text {hol }}^{\omega}(X)\right\}
$$

which in combination with the above description of generators of $H^{n-1}\left(X, \Omega^{n-1}\right)$ yields the first statement.

To prove the second assertion note that the $\mathcal{O}(X)$-module homomorphism of sheafs

$$
\mathcal{O}(X)^{N} \rightarrow T X, \quad\left(f_{1}, f_{2}, \ldots, f_{N}\right) \mapsto \sum_{i=1}^{N} f_{i} \delta_{i}
$$

and, therefore (because of the isomorphism $\nu \rightarrow \iota_{\nu}(\omega)$ ) the homomorphism

$$
\mathcal{O}(X)^{N} \rightarrow \Omega^{n-1}, \quad\left(f_{1}, f_{2}, \ldots, f_{N}\right) \mapsto \omega_{\sum_{i=1}^{N} f_{i} \delta_{i}}
$$

are surjective. Since $X$ is Stein, the Cartan Theorem B implies the surjectivity on the level of global sections. Together with Lemma 4.2 this implies the second assertion.

In combination with Lemma 4.1 this implies the following.

4.5. Corollary. For a Stein manifold $X$ with the volume density property we have finitely many vector fields $\delta_{1}, \delta_{2}, \ldots, \delta_{N} \in \mathrm{IVF}_{\text {hol }}^{\omega}(X)$ for which

$$
F_{X}=\operatorname{Span}\left\{\delta_{i}(\operatorname{Hol}(X)) \mid i=1, \ldots, N\right\} .
$$

4.6. Lemma. If $X$ is Stein, then $F_{X}$ is a closed linear subspace of $\operatorname{Hol}(X)$ and thus a Frechet space. Moreover, $\operatorname{Hol}(X) / F_{X}$ is isomorphic to $H^{n}(X, \mathbb{C})$. 
Proof. Since $X$ is Stein the inclusion of the holomorphic de Rham complex into the complex-valued de Rham $C^{\infty}$-complex

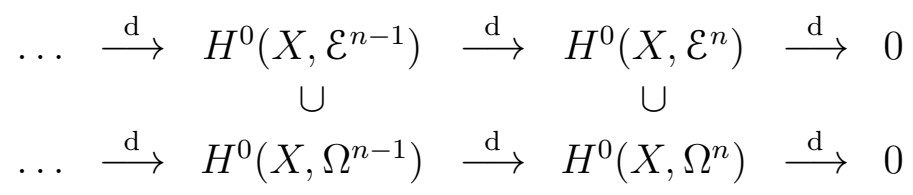

is a quasi-isomorphism, i.e. the complex cohomology of $X$ can be computed via the lower complex. By de Rham's theorem the boundaries $\mathrm{d}\left(H^{0}\left(X, \mathcal{E}^{n-1}\right)\right)$ are exactly the $n$-forms whose integral over any real $n$-dimensional cycle is zero (e.g., see [36]) and, therefore, $\mathrm{d}\left(H^{0}\left(X, \Omega^{n-1}\right)\right)$ consists exactly of the holomorphic $n$-forms which give zero when integrated over any real $n$-dimensional cycle. This condition is closed in the compact-open topology, which proves the first assertion. The second one follows from $H^{n}(X, \mathbb{C})=H^{0}\left(X, \mathcal{E}^{n}\right) / \mathrm{d}\left(H^{0}\left(X, \mathcal{E}^{n-1}\right)\right) \cong H^{0}\left(X, \Omega^{n}\right) / \mathrm{d}\left(H^{0}\left(X, \Omega^{n-1}\right)\right) \cong \operatorname{Hol}(X) / F_{X}$, where the last isomorphism is induced by $\varepsilon$ from lemma 4.4 .

4.7. Grothendieck's tensor products. For convenience of readers we remind some facts from GrothendiECK's theory of nuclear vector spaces which can be found in [61] or [69]. Denote by $E, E_{i}, F, F_{i}$, and $H$ locally convex Hausdorff topological vector spaces. Grothendieck introduced two different completions of the algebraic tensor product $E_{1} \otimes E_{2}$, denoted by $E_{1} \hat{\otimes}_{\pi} E_{2}$ and $E_{1} \hat{\otimes}_{\varepsilon} E_{2}$. The crucial properties of these completions we are going to exploit are the following. For linear continuous isomorphisms into (i.e embeddings) $E_{1} \rightarrow F_{1}$ and $E_{2} \rightarrow E_{2}$ the induced linear map $E_{1} \hat{\otimes}_{\varepsilon} E_{2} \rightarrow F_{1} \hat{\otimes}_{\varepsilon} F_{2}$ is also an isomorphism into [69, Proposition 43.7]. In the case of metrizable $E_{1}$ and $E_{2}$ and linear continuous surjective maps $E_{1} \rightarrow F_{1}$ and $E_{2} \rightarrow E_{2}$ the induced linear map $E_{1} \hat{\otimes}_{\pi} E_{2} \rightarrow F_{1} \hat{\otimes}_{\pi} F_{2}$ is also a surjection [69, Proposition 43.9].

A space $E$ is called nuclear if for any other locally convex topological vector space $F$ the completions $E \hat{\otimes}_{\varepsilon} F$ and $E \hat{\otimes}_{\pi} F$ coincide which allows us to omit indices $\pi$ and $\varepsilon$ in tensor products when appropriate. Nuclear spaces possess nice properties: their subspaces and quotients with respect to closed subspaces are again nuclear [69, Proposition 50.1]. Furthermore, the above claims about surjections and embeddings in combination with [69, Exercise 43.2$]^{9}$ imply that the GrothendieCK's tensor product preserves (short) exact sequences of metrizable nuclear spaces. That is, $(E / H) \hat{\otimes} F=(E \hat{\otimes} F) /(H \hat{\otimes} F)$ where $H$ is a closed subspace of the nuclear space $E$.

4.8. Example. Let $X$ (resp. $Y$ ) be a closed Stein submanifold of $\mathbb{C}^{n}\left(\right.$ resp. $\left.\mathbb{C}^{m}\right)$, i.e we have the natural surjections $\varphi_{X}: \operatorname{Hol}\left(\mathbb{C}^{n}\right) \rightarrow \operatorname{Hol}(X)$ and $\varphi_{Y}: \operatorname{Hol}\left(\mathbb{C}^{m}\right) \rightarrow \operatorname{Hol}(Y)$. The spaces of holomorphic functions on Euclidean spaces are known to be nuclear [69, Corollary, p. 530] and, therefore, their quotients $\operatorname{Hol}(X)$ and $\operatorname{Hol}(Y)$ are also nuclear. Furthermore $\operatorname{Hol}\left(\mathbb{C}^{n}\right) \hat{\otimes} \operatorname{Hol}\left(\mathbb{C}^{m}\right)$ is naturally isomorphic to $\operatorname{Hol}\left(\mathbb{C}^{n+m}\right)$ 69, Theorem 51.6] and the induced surjective linear map $\varphi_{X} \hat{\otimes} \varphi_{Y}: \operatorname{Hol}\left(\mathbb{C}^{n+m}\right) \rightarrow \operatorname{Hol}(X) \hat{\otimes} \operatorname{Hol}(Y)$

\footnotetext{
${ }^{9}$ Using this exercise one can show that for surjective maps $\varphi_{1}: E_{1} \rightarrow F_{1}$ and $\varphi_{2}: E_{2} \rightarrow E_{2}$ the induced linear map $\varphi_{1} \hat{\otimes} \varphi_{2}: E_{1} \hat{\otimes} E_{2} \rightarrow F_{1} \hat{\otimes} F_{2}$ has $\operatorname{Ker} \varphi_{1} \hat{\otimes} \varphi_{2}=\operatorname{Ker} \varphi_{1} \hat{\otimes} E_{2}+E_{1} \hat{\otimes} \operatorname{Ker} \varphi_{2}$.
} 
has the kernel generated by functions that vanish either on $X \times \mathbb{C}^{m}$ or on $\mathbb{C}^{n} \times Y$. Thus this kernel coincides with the defining ideal of $X \times Y$ in $\mathbb{C}^{n+m}$ and $\operatorname{Hol}(X) \hat{\otimes} \operatorname{Hol}(Y)$ is naturally isomorphic to $\operatorname{Hol}(X \times Y)$.

We present the proof of the next simple fact because of the lack of references.

4.9. Lemma. Let $F_{i}$ be a closed subspace of a complete metrizable nuclear space $E_{i}$ for $i=1,2$. Then $I:=\left(F_{1} \hat{\otimes} E_{2}\right) \cap\left(E_{1} \hat{\otimes} F_{2}\right)$ coincides with $F_{1} \hat{\otimes} F_{2}$.

Proof. Set $Q_{i}=E_{i} / F_{i}$. Then the kernel of the natural linear map $E_{1} \hat{\otimes} E_{2} \rightarrow Q_{1} \hat{\otimes} Q_{2}$ coincides with $F_{1} \hat{\otimes} E_{2}+E_{1} \hat{\otimes} F_{2}$. In particular, $Q_{1} \hat{\otimes} Q_{2}$ is naturally isomorphic to the quotinet of $\left(E_{1} \hat{\otimes} E_{2}\right) /\left(E_{1} \hat{\otimes} F_{2}\right)=E_{1} \hat{\otimes} Q_{2}$ with respect to $M:=\left(F_{1} \hat{\otimes} E_{2}\right) / I$ which implies that $M$ coincides with the subspace $F_{1} \hat{\otimes} Q_{2}$ of $E_{1} \hat{\otimes} Q_{2}$ since $\left(E_{1} \hat{\otimes} Q_{2}\right) /\left(F_{1} \hat{\otimes} Q_{2}\right)=$ $Q_{1} \hat{\otimes} Q_{2}$. Note also that $F_{1} \hat{\otimes} Q_{2} \simeq\left(F_{1} \hat{\otimes} E_{2}\right) /\left(F_{1} \hat{\otimes} F_{2}\right)$. Since $I \supset F_{1} \hat{\otimes} F_{2}$ and $M:=$ $\left(F_{1} \hat{\otimes} E_{2}\right) / I$ we must have $I=F_{1} \hat{\otimes} F_{2}$.

We need an extra product $E \varepsilon F$ of locally convex topological vector spaces introduced by SCHWARTz [62]. In general $E \hat{\otimes}_{\varepsilon} F$ is a subspace of $E \varepsilon F$. However we have equality $E \hat{\otimes}_{\varepsilon} F=E \varepsilon F$ provided that both $E$ and $F$ are complete and have the approximation property (see, [62, Corollary 1, p. 47]). The latter means, say for $E$, that the identical operator on $E$ belongs to the closure of operators $E \rightarrow E$ of finite rank in the topology $\mathcal{L}_{c}(E, E)$ of uniform convergence on convex compact subsets of $E$ (see, [62, Definition, p. 5]). For any nuclear space $E$ the identical operator can be always approximated by operators of finite rank. Thus the result of Bungart [14, Proposition 9.2] about $\varepsilon$-product can be reformulated in the special case of nuclear spaces as the following.

4.10. Proposition. Let $X$ be a Stein manifold and $E$ be a complete nuclear space. Then $\operatorname{Hol}(X) \hat{\otimes}_{\varepsilon} E$ coincides with the space of weakly holomorphic E-valued functions on $X$ (i.e. E-valued functions $f$ on $X$ such that if for any continuous linear functional $T$ on $E$ the function $T \circ f$ is holomorphic on $X)$.

4.11. Convention. For Stein manifolds $X$ and $Y$ we consider holomorphic vector fields on $X \times Y$ tangent to the fibers of the natural projection $X \times Y \rightarrow X$ (resp. $X \times Y \rightarrow Y$ ) and we call them vertical (resp. horizontal) fields. Every holomorphic vector field $\delta$ and $X$ (resp. $\mu$ on $Y$ ) generates the natural horizontal (resp. vertical) vector field on $X \times Y$ which by abuse of notation will be denoted by the same symbol.

4.12. Lemma. Let Notation 4.3 and Convention 4.11 hold, and let $\delta_{1}, \delta_{2}, \ldots, \delta_{N} \in$ $\operatorname{IVF}_{\text {hol }}^{\omega}(X)$ be as in Corollary 4.5. Then for every $f \in F_{X} \hat{\otimes} \operatorname{Hol}(Y)$ there exist $\bar{b}=$ $\left(b_{1}, \ldots, b_{N}\right) \in \operatorname{Hol}(X \times Y)^{N}$ such that $f=\delta_{1}\left(b_{1}\right)+\delta_{2}\left(b_{2}\right)+\ldots+\delta_{N}\left(b_{N}\right) 10$.

Proof. By Lemma 4.6 the map $\Theta: \operatorname{Hol}(X)^{N} \rightarrow F_{X}$ given by $\left(a_{1}, a_{2}, \ldots, a_{N}\right) \mapsto$ $\sum_{i=1}^{N} \delta_{i}\left(a_{i}\right)$ is a linear surjection of Frechet spaces. By GRothendiECK's theorem

\footnotetext{
${ }^{10}$ In this formula each $\delta_{j}$ is already a horizontal field on $X \times Y$.
} 
the linear map

$$
\Theta \hat{\otimes} \operatorname{id}_{Y}:(\operatorname{Hol}(X))^{N} \hat{\otimes} \operatorname{Hol}(Y) \cong(\operatorname{Hol}(X \times Y))^{N} \rightarrow F_{X} \hat{\otimes} \operatorname{Hol}(Y)
$$

is surjective which implies the desired conclusion

4.13. Remark. (1) The proof of existence of $\bar{b}$ from Lemma 4.12 can be also extracted from another nontrivial fact - the theorem of MichaEL (e.g., see [40, p. 183-186] and [1, Corollary 17.67]) that states that for every continuous surjective linear map of Frechet spaces there exists a homogeneous continuous section.

(2) The same argument implies that for every $f \in F_{X} \hat{\otimes} F_{Y}$ there is $\bar{b}=\left(b_{1}, \ldots, b_{N}\right) \in$ $\left(\operatorname{Hol}(X) \hat{\otimes} F_{Y}\right)^{N}$ for which $f=\delta_{1}\left(b_{1}\right)+\ldots+\delta_{N}\left(b_{N}\right)$.

4.14. Proposition. For every vector field $\mu \in \operatorname{IVF}_{\text {hol }}^{\omega}(Y)$ and each $f \in F_{X} \hat{\otimes} \operatorname{Hol}(Y)$ there is a vector field $\alpha$ from the closure of $\mathrm{Lie}_{\mathrm{hol}}^{\omega_{X} \times \omega_{Y}}(X \times Y)$ (in the compact-open topology) such that the field $f \mu-\alpha$ is a horizontal vector field on $X \times Y$.

Proof. By Lemma $4.12 f=\delta_{1}\left(b_{1}\right)+\delta_{2}\left(b_{2}\right)+\ldots+\delta_{N}\left(b_{N}\right)$. Fix a closed embedding $\iota: Y \rightarrow \mathbb{C}_{y_{1}, \ldots, y_{m}}^{m}$ and denote a monomial $y_{1}^{k_{1}} \cdots y_{m}^{k_{m}}$ by $y^{k}$, i.e. $k$ is the multi-index $\left(k_{1}, \ldots, k_{m}\right)$ with norm $|k|=k_{1}+\ldots+k_{m}$. Then $b_{i}$ can be presented as $b_{i}=\sum_{k} b_{i, k} y^{k}$ where $b_{i, k} \in \operatorname{Hol}(X)$ and the sum converges uniformly on compacts of $X \times Y$. Let us show that the desired limit can be given by the formula

$$
\alpha=\lim _{M \rightarrow \infty} \sum_{|k|<M} \sum_{i=1}^{N}\left[y^{k} \delta_{i}, b_{i, k} \mu\right] .
$$

Indeed, since $y^{k}$ (resp. $b_{i, k}$ ) is in the kernel of $\delta_{i}$ (resp. $\mu$ ) the involved vector fields are completely integrable and of $\left(\omega_{X} \times \omega_{Y}\right)$-divergence zero. Moreover

$$
\left[y^{k} \delta_{i}, b_{i, k} \mu\right]=y^{k} \delta_{i}\left(b_{i, k}\right) \mu-b_{i, k} \mu\left(y^{k}\right) \delta_{i} \text {. }
$$

Thus

$$
\begin{gathered}
\sum_{|k|<M} \sum_{i=1}^{N}\left[y^{k} \delta_{i}, b_{i, k} \mu\right]=\left(\sum_{|k|<M} \sum_{i=1}^{N} y^{k} \delta_{i}\left(b_{i, k}\right)\right) \mu-\sum_{i=1}^{N}\left(\sum_{|k|<M} b_{i, k} \mu\left(y^{k}\right)\right) \delta_{i}= \\
=\left(\sum_{i=1}^{N} \delta_{i}\left(\sum_{|k|<M} b_{i, k} y^{k}\right)\right) \mu-\sum_{i=1}^{N} \mu\left(\sum_{|k|<M} b_{i, k} y^{k}\right) \delta_{i} .
\end{gathered}
$$

By the Weierstrass theorem about differentation of convergent power series we can send $M \rightarrow \infty$ and obtain

$$
\alpha=f \mu-\sum_{i=1}^{N} \mu\left(b^{i}\right) \delta_{i}
$$

which yields the desired conclusion. 
4.15. Proof of Theorem 13, Let completely integrable holomorphic vector fields $\mu_{1}, \ldots, \mu_{M}$ on $Y$ play the same role as $\delta_{1}, \ldots, \delta_{N}$ from Corollary 4.5 play for $X$. In particular, $\mu_{1}, \ldots, \mu_{M}$ generate the tangent vector space at any point of $Y$. Hence a simple application of the Cartan theorem B implies that every vector field on $X \times Y$ is of form

$$
\gamma=\sum_{i=1}^{M} f_{i} \mu_{i}+\sum_{j=1}^{N} g_{j} \delta_{j}
$$

where $f_{i}$ and $g_{j}$ are holomorphic functions on $X \times Y$. We suppose further that $\gamma$ is of $\omega$-divergence zero where $\omega=\omega_{X} \times \omega_{Y}$. Recall that for every holomorphic vector field $\nu$ of $\omega$-divergence zero we have $\operatorname{div}_{\omega} f \nu=\nu(f)$ for every holomorphic functions $f$. Since $\mu_{i}$ and $\delta_{j}$ have $\omega$-divergence zero, the divergence of $\gamma$ coincides with

$$
\operatorname{div}_{\omega} \gamma=\sum_{i=1}^{M} \mu_{i}\left(f_{i}\right)+\sum_{j=1}^{N} \delta_{j}\left(g_{j}\right)=0 .
$$

Note that the first (resp. second) summand can be viewed as a weakly holomorphic function on $X$ (resp. Y) with values in $F_{Y}$ (resp. $\left.F_{X}\right)$.

Thus by BUNGART's theorem (Proposition 4.10) we have

$$
f:=\sum_{i=1}^{M} \mu_{i}\left(f_{i}\right) \in \operatorname{Hol}(X) \hat{\otimes} F_{Y} \text { and } \sum_{j=1}^{N} \delta_{j}\left(g_{j}\right) \in F_{X} \hat{\otimes} \operatorname{Hol}(Y) .
$$

By Lemma $4.9 \sum_{i=1}^{M} \mu_{i}\left(f_{i}\right) \in F_{X} \hat{\otimes} F_{Y}$. Furthermore, by Remark 4.13 (2) there exist $h_{1}, \ldots, h_{M} \in F_{X} \hat{\otimes} \operatorname{Hol}(Y)$ for which

$$
f=\mu_{1}\left(h_{1}\right)+\ldots \mu_{M}\left(h_{M}\right) .
$$

By Proposition 4.14 there exists a vector field $\nu \in \operatorname{Lie}_{\text {hol }}^{\omega}(X \times Y)$ of form

$$
\nu=\sum_{i=1}^{M} h_{i} \mu_{i}+\sum_{j=1}^{N} e_{j} \delta_{j}
$$

Subtracting $\nu$ from $\gamma$ we can suppose from the beginning that

$$
\operatorname{div}_{\omega} \sum_{i=1}^{M} f_{i} \mu_{i}=\sum_{i=1}^{M} \mu_{i}\left(f_{i}\right)=0
$$

which implies that

$$
\operatorname{div}_{\omega} \sum_{i=1}^{N} g_{i} \delta_{i}=\sum_{i=1}^{N} \delta_{i}\left(g_{i}\right)=0
$$

because $\operatorname{div}_{\omega} \gamma=0$. Since each $\mu_{i}$ is a vertical vector field on $X \times Y$ this means that the restriction of $\sum_{i=1}^{M} f_{i} \mu_{i}$ to any fiber $x \times Y$ is of $\omega_{Y}$-divergence zero and therefore it belongs to $\operatorname{Li}_{\text {hol }}^{\omega_{Y}}(x \times Y)$ by the assumption of the theorem. That is, $\sum_{i=1}^{M} f_{i} \mu_{i}$ can be viewed as a weakly holomorphic function on $X$ with values in $\operatorname{Lie}_{\text {hol }}^{\omega_{Y}}(Y)$. By BungarT's theorem (Proposition 4.10) it belongs to $\operatorname{Hol}(X) \hat{\otimes} \operatorname{Lie}_{\text {hol }}^{\omega_{Y}}(Y)$ which is a subspace of 
$\operatorname{Lie}_{\text {hol }}^{\omega}(X \times Y)$. The same argument implies that $\sum_{i=1}^{N} g_{i} \delta_{i} \in \operatorname{Lie}_{\text {hol }}^{\omega}(X \times Y)$ and thus $\gamma \in$ $\operatorname{Lie}_{\text {hol }}^{\omega}(X \times Y)$ which is the desired conclusion.

\section{Preliminary facts about Foliation}

A more detailed exposition of the results from this section can be found in [11], [12], or $[13$.

5.1. Definition. (1) A foliation $\mathcal{F}$ on a smooth complex surface $\bar{X}$ is given by an open covering $\left\{U_{j}\right\}$ of $\bar{X}$ and holomorphic vector fields $\nu_{j} \in H^{0}\left(U_{j}, T \bar{X}\right)$ with isolated zeros such that

$$
\nu_{i}=g_{i j} \nu_{j} \text { on } U_{i} \cap U_{j}
$$

for invertible holomorphic functions $g_{i j} \in H^{0}\left(U_{i} \cap U_{j}, \mathcal{O}_{X}^{*}\right)$ where $\mathcal{O}_{X}^{*}$ is the sheaf of invertible functions. Gluing orbits of $\left\{\nu_{j}\right\}$ one gets leaves of the foliation $\mathcal{F}$. The singular set $\operatorname{Sing}(\mathcal{F})$ is the discrete subset of $\bar{X}$ whose intersection with each $U_{j}$ coincides with with zeros of $\nu_{j}$. The cocycle $\left\{g_{i j}\right\}$ define a holomorphic line bundle $K_{\mathcal{F}}$ which is called the canonical bundle of the foliation $\mathcal{F}$.

(2) This definition can be extended to the case of $\bar{X}$ with quotient singularities only where $\mathcal{F}$ defined as a foliation on $\bar{X} \backslash \operatorname{Sing}(\bar{X})$. We require additionally that

$$
\operatorname{Sing}(\bar{X}) \cap \operatorname{Sing}(\mathcal{F})=\varnothing \text {. }
$$

That is, a singular point $p$ of $\bar{X}$ is locally of form $\mathbb{B}^{2} / \mathbb{Z}_{k}$ where $\mathbb{B}^{2}$ is a ball in $\mathbb{C}^{2}$ equipped with a linear $\mathbb{Z}_{k}$-action. In particular, the foliation can be lifted to $\mathbb{B}^{2} \backslash\{(0,0)\}$ and the requirement is that it can extended to a foliation on $\mathbb{B}^{2}$ with a non-vanishing associated vector field $\nu$ (and this must be true for any singular point of $\bar{X}$ ).

Then $K_{\mathcal{F}}$ on $\bar{X}$ is the direct image of the canonical bundle on $\bar{X} \backslash \operatorname{Sing}(\bar{X})$ under the inclusion morphism $\bar{X} \backslash \operatorname{Sing}(\bar{X}) \hookrightarrow \bar{X}$ (in this situation $K_{\mathcal{F}}$ is not a bundle but only a $\mathbb{Q}$-bundle).

(3) Foliation $\mathcal{F}$ is called nef if $K_{\mathcal{F}}$ is nef.

(4) A singularity $p \in \operatorname{Sing}(\mathcal{F})$ is reduced if the linear part of the corresponding vector field at $p$ has eigenvalues $\lambda_{1}, \lambda_{2}$ such that either they are nonzero and $\lambda_{1} / \lambda_{2} \notin \mathbb{Q}_{+}$or $\lambda_{1} \neq 0=\lambda_{2}$. The foliation $\mathcal{F}$ is called reduced if all of its singularities are reduced.

(5) The Kodaira dimension $\operatorname{kod}(\mathcal{F})$ of a reduced foliation $\mathcal{F}$ on a projective surface $\bar{X}$ is the Kodaira-Iitaka dimension of its canonical bundle $K_{\mathcal{F}} \in \operatorname{Pic}(\bar{X}) \otimes \mathbb{Q}$. That is,

$$
\operatorname{kod}(\mathcal{F})=\lim \sup _{n \rightarrow+\infty} \frac{\log \operatorname{dim} H^{0}\left(\bar{X}, K_{\mathcal{F}}^{\otimes n}\right)}{\log n}
$$

We shall study foliations associated with completely integrable holomorphic vector fields on a Stein surface $X$ and the following result of Suzuki [65], [66] is very important.

Theorem 14. Let $\mathcal{F}$ be a foliation on a normal Stein surface $X$.

(1) If all leaves of $\mathcal{F}$ are properly embedded in $X \backslash \operatorname{Sing}(\mathcal{F})$ then there is a nonconstant meromorphic first integral of $\mathcal{F}$ on $X$. 
(2) Furthermore, if the general leaf of $\mathcal{F}$ is isomorphic to $\mathbb{C}^{*}$ (we shall call below such foliations of $\mathbb{C}^{*}$-type) then every leaf is closed in $X \backslash \operatorname{Sing}(\mathcal{F})$ and therefore there is a meromorphic first integral as in (1).

For such a normal affine algebraic surface $X$ the study of the foliation $\mathcal{F}$ would be much simpler when this first integral were a rational function. We need to consider the situation when this first integral is not rational.

First note that a foliation associated with an algebraic vector fields on $X$ can be extended to a completion $\bar{X}$ of $X$ and we can deal with a projective normal surface. Then we have the following result of SEIDENBERG (e.g., see [11]).

Theorem 15. Performing a sequence of blow-ups $\tilde{X} \rightarrow \bar{X}$ we obtain a lifted foliation $\tilde{\mathcal{F}}$ on $\tilde{X}$ which is reduced.

Thus from now on we shall work with reduced foliations on projective surfaces. MiYaOKA and Shepherd-BARron (e.g., see [12]) established the following.

Theorem 16. If $\mathcal{F}$ is a reduced foliation on a projective surface $\bar{X}$ with at most cyclic quotient singularities then $K_{\mathcal{F}}$ is pseudoeffective if and only if $\mathcal{F}$ is not a rational foliation (i.e. its general fiber is a rational curve in $\mathbb{C P}^{1}$ ).

The Zariski-Fujita decomposition implies that every pseudoeffective $\mathbb{Q}$-bundle is the sum of a nef $\mathbb{Q}$-bundle and a negative part for which the associated divisor can be contracted. This is a basis for the next fact (MCQUILLAN's contraction) (e.g., see [11] or [12]).

Theorem 17. Let $\mathcal{F}$ be a non-rational reduced foliation on a projective surface $\bar{X}$ with at most cyclic quotient singularities. Then there exists a birational morphism $(\bar{X}, \mathcal{F}) \rightarrow$ $\left(\bar{X}^{\prime}, \mathcal{F}^{\prime}\right)$ such that $\bar{X}^{\prime}$ is still projective with at most cyclic quotient singularities, $\mathcal{F}^{\prime}$ is still reduced, and $K_{\mathcal{F}^{\prime}}$ is nef.

5.2. Remark. ([13, Section 3] or [12, page 10]) Contraction of $\bar{X}$ to $\bar{X}^{\prime}$ is a sequence of blowing down of rational curves such that each of them is invariant with respect to the consequent induced foliation and the restriction of the canonical bundle of the foliation to the curve is negative. Every of these curves $F$ contains exactly one singularity $p$ of the foliation which is automatically a regular point of the surface. Furthermore, $F$ is contracted to a point which is a regular point of the induced foliation on the resulting surface (but not in general a regular point of the surface).

The reduced foliation generated by a completely integrable algebraic vector field on $X$ admits a lot of entire tangent curves $\mathbb{C} \rightarrow \bar{X}$. If $\mathcal{F}$ has no rational first integral, then such general curve is Zariski dense in $\bar{X}$ (Darboux's theorem) and another result of MCQuillan says the following (see [54, Sections IV and V] or [11, Chapter 9, Theorems 1 and 4, Corollary 1].

Theorem 18. Let $\mathcal{F}$ be a reduced foliation on a smooth projective surface $\bar{X}$ such that $\mathcal{F}$ possesses a tangent nonconstant entire curve that is Zariksi dense in $\bar{X}$. Then the Kodaira dimension $\operatorname{kod}(\mathcal{F})$ is either 0 or 1. 
(1) Furthermore, if $\operatorname{kod}(\mathcal{F})=1$ then either

(1a) $\mathcal{F}$ is a Riccati foliation, i.e. there exists a fibration $f: \bar{X} \rightarrow B$ whose general fiber is a rational curve transverse to $\mathcal{F}$ or

(1b) $\mathcal{F}$ is a Turbulent foliation, i.e. there exists a fibration $f: \bar{X} \rightarrow B$ whose general fiber is an elliptic curve transverse to $\mathcal{F}$.

(2) If $\operatorname{kod}(\mathcal{F})=0$ and $\bar{X} \rightarrow \bar{X}^{\prime}$ is the MCQUILLAN's contraction to a nef reduced foliation $\mathcal{F}^{\prime}$ on $\bar{X}^{\prime}$ then there exists a finite covering $r: Y \rightarrow \bar{X}^{\prime}$ such that

(2a) $Y$ is smooth and $r$ is ramified only over the quotient singularities of $\bar{X}^{\prime}$.

(2b) The canonical bundle $K_{\mathcal{G}}$ of the lifted foliation $\mathcal{G}=r^{*}\left(\mathcal{F}^{\prime}\right)$ is trivial, i.e. $K_{\mathcal{G}}=$ $\mathcal{O}_{Y}$, and so $\mathcal{G}$ is generated by a global holomorphic vector field with isolated zeros only.

6. Brunella's Construction: the CASE of $\operatorname{kod}(\mathcal{F})=1$.

In fact BRUNELLA proved more (Lemmas 1 and 2 in [13]).

6.1. Proposition. (1) Case (1b) in Theorem 18 cannot hold, i.e. when $\operatorname{kod}(\mathcal{F})=1$ for a reduced foliation without a rational first integral then it is a Riccati foliation

(2) Contracting curves in the fibers of $f: \bar{X} \rightarrow B$ for the Riccati foliation on a smooth surface $\bar{X}$ one can suppose that each fiber of $f$ belongs to one of five standard types and there is always at least one singular fiber of $f$ of so-called types (e) or (d) (see [13, Lemma 2]) that consists of a union of leaves of the foliation $\mathcal{F}$ (it will be denoted below by $\left.F_{\infty}\right)$.

Next we have the following (Lemmas 3-5 in [13]).

6.2. Proposition. Let $\nu$ be a completely integrable algebraic vector field on a rational affine algebraic surface $X$ and $\pi: \hat{X} \rightarrow X$ be the resolution of the singularities of $\nu$ (i.e. the lift $\hat{\nu}$ of $\nu$ is a completely integrable vector field on $\hat{X}$ ). Suppose that the foliation $\mathcal{F}$ generated by $\hat{\nu}$ on the completion $\bar{X}$ of $\hat{X}$ is reduced and has no rational first integral. Let $f: \bar{X} \rightarrow B$ be the corresponding (rational) Riccati fibration (note that $B \simeq \mathbb{C P}^{1}$ since $\bar{X}$ is rational). Then

(1) the vector field $\hat{\nu}$ preserves $\left.f\right|_{\hat{X}}$ (i.e. its flow sends fibers into fibers) or in other words $\hat{\nu}$ is a lift of a completely integrable vector field $\nu_{0}$ on $f(\hat{X}) \subset B$;

(2) the fiber $F_{\infty}$ is contained in the divisor $D=\bar{X} \backslash \hat{X}$ and in particular the exceptional divisor $E$ of $\pi$ is disjoint from $F_{\infty}$;

(3) contracting $E$ we get from $f$ a regular function $P$ on $X$ whose general fibers are either $\mathbb{C}$ or $\mathbb{C}^{*}$ (i.e. again the flow of $\nu$ maps each fiber of $P$ onto a fiber of $P$ ).

In fact for foliations of $\mathbb{C}^{*}$-type the same conclusion can be made even without assumption that $\operatorname{kod}(\mathcal{F})=1$. In order to demonstrate it we need some facts from another BRUNELLA's paper [10] where he used a slightly different terminology. Let $L$ be a leaf of the foliation $\mathcal{F}$ on $X$ and $L_{0}$ be a Riemann surface isomorphic to $\{z \in \mathbb{C}|0 \leq r<| z \mid \leq 1\}$ which is properly embedded into $L$. Then $L_{0}$ is called a planar isolated end of $L$. This end is called transcendental if the set $\bar{L}_{0} \backslash L_{0}$ consists of more than one point where $\bar{L}_{0}$ is the closure of $L_{0}$ in $\bar{X}$. Fibration $\mathcal{F}$ is called (in [10]) 
$P$-complete for some regular function $P$ on $X$ if there exists a finite set $Q$ such that for every $t \in \mathbb{C} \backslash Q$ the fiber $P^{-1}(t)$ is transversal to $\mathcal{F}$ and there is a neighborhood $U_{t} \subset \mathbb{C}$ of $t$ for which $\left.P\right|_{P^{-1}\left(U_{t}\right)}$ is a fibration and $\left.\mathcal{F}\right|_{P^{-1}\left(U_{t}\right)}$ define a local trivialization of it.

The next fact is the main result in [10].

6.3. Proposition. Let $X$ be a smooth rational affine surface with an SNC-completion $\bar{X}$ and $\bar{D}=\bar{X} \backslash X$. Suppose that for any other $S N C$-completion $\overline{\bar{X}}$ of $X$ that dominates $\bar{X}$ (i.e. there is a morphism $\pi: \overline{\bar{X}} \rightarrow \bar{X}$ identical on $X$ ) the following is true.

(i) There is a Kahler metric on $\overline{\bar{X}}$ such that the restriction of the associated 2-form to $X$ is exact.

(ii) Let $\Gamma$ be the dual graph of the divisor $\bar{D}$ and let $\Gamma$ have no linear $(-1)$-vertices. Suppose also that for any branch point $b$ of $\Gamma$ such that the subgraph $\Gamma \ominus b$ contains two connected components (among others), each of which is contractible to a (-2)-vertex, the weight of $b$ is at most -2 (note that this condition will hold automatically for the dual graph of $\overline{\bar{D}}=\overline{\bar{X}} \backslash X)$.

Then for any foliation $\mathcal{F}$ generated by a regular vector field on $X$, which possesses also a transcendental planar isolated end, there exists a regular function $P \in \mathbb{C}[X]$ with general fibers isomorphic either to $\mathbb{C}$ or to $\mathbb{C}^{*}$ such that $\mathcal{F}$ is $P$-complete.

6.4. Remark. (1) This regular function $P$ yields, of course, a Riccati foliation which is mentioned by BRunella [10, p. 1241]. He mentions also in [13] that the construction from [10, pp. 1241-1243] implies that one of singular fibers of $P$ is of type (d).

(2) Suppose that $\mathcal{F}$ is a foliation of $\mathbb{C}^{*}$-type that has no rational first integral. Then general leaves are isomorphic to $\mathbb{C}^{*}$, they are Zariski dense in $\bar{X}$ and properly embedded into the complement to singularities by Theorem 14. This implies the existence of a transcendental planar isolated end, i.e. Proposition 6.3 is applicable to foliations of $\mathbb{C}^{*}$-type generated by regular vector fields.

(3) In [10] this Proposition 6.3 is proven only for $X=\mathbb{C}^{2}$. However the analysis of the proof shows that it is valid for any smooth rational affine surface $X$ satisfying condition (i) and (ii). Condition (i) (which is obviously true for $\mathbb{C}^{2}$ since the second cohomology of $\mathbb{C}^{2}$ is trivial) is used in Lemma 3 in 10 . Condition (ii) is also true for $\mathbb{C}^{2}$ and used in the proof of Lemma 8 in [10]. The next (certainly well-known) fact says more about condition (i).

6.5. Proposition. Condition (i) from Proposition 6.3 is automatic for every affine algebraic manifold $X$.

Proof. We can choose an SNC-completion $\bar{X}$ of $X$ so that $\bar{X} \backslash X$ is a support of an ample divisor (for a two-dimensional $X$ the facts that $X$ is affine and that $\bar{X} \backslash X$ is the support of an ample divisor are equivalent). Then this divisor generates an embedding $\bar{X} \hookrightarrow \mathbb{P}^{n}=: \bar{Y}$ such that for a hyperplane $H \subset \bar{Y}$ the Euclidean space $\bar{Y} \backslash H=: Y \simeq \mathbb{C}^{n}$ contains $X$ as a closed submanifold. The birational morphism $\pi: \overline{\bar{X}} \rightarrow \bar{X}$ may be viewed as the blow-up of an ideal sheaf $\mathcal{I}$ [39, Theorem II.7.17]. Consider the ideal 
sheaf on $\bar{Y}$ generated by $\mathcal{I}$ and the defining equations of $\bar{X}$. The blow-up with respect to this new sheaf yields a birational morphism $\tau: \overline{\bar{Y}} \rightarrow \bar{Y}$ which is identical over $Y$, i.e. $\overline{\bar{Y}}$ is another completion of $Y$. Furthermore, $\pi$ is the restriction of $\tau$ to the proper transform of $\bar{X}$ [39, Corollary II.7.15]. Since $\overline{\bar{Y}}$ is projective it is a subvariety of $\mathbb{P}^{N}$ which is equipped with a closed 2-form $\omega$ as a Kahler manifold. The restriction of $\omega$ to $Y$ is an exact form by de Rham's theorem because the second cohomology of $Y$ is trivial. Thus its restriction to $X$ is also exact which is the desired conclusion.

6.6. Proposition. (cf. [13, Proposition 3]) Let $\nu$ be a completely integrable algebraic vector field of type $\mathbb{C}^{*}$ on a smooth affine rational surface $X$ satisfying condition (ii) from Proposition 6.3. Then either $\nu$ possesses a rational first integral or for some regular function $P$ on $X$ with general fibers $\mathbb{C}$ or $\mathbb{C}^{*}$ the flow of $\nu$ transforms fibers of $P$ into fibers of $P$.

Proof. By Theorem 14 the leaves of the associated foliation $\mathcal{F}$ are properly embedded into $X \backslash \operatorname{Sing}(\mathcal{F})$ and therefore there is a meromorphic first integral. Assume that it is not rational. Then there is at least one transcendental end in $X$ (otherwise all leaves are algebraic and the first integral is rational by the Darboux's theorem). Now Proposition 6.3 and Remark 6.4 imply the existence of a Riccati fibration $P$ with general fibers $\mathbb{C}$ or $\mathbb{C}^{*}$ such that the fiber over $\infty$ is of type $(\mathrm{d})$ which is the only thing needed for validity of Proposition 6.2 whose proof in [13] does not use other properties of foliations with $\operatorname{kod}(\mathcal{F})=1$.

7. Brunella's Construction: the CASE of $\operatorname{kod}(\mathcal{F})=0$.

Thus we can consider only completely integrable algebraic fields of type $\mathbb{C}$ for which most of orbits are isomorphic to $\mathbb{C}$. For a smooth affine surface $X$ with a completely integrable algebraic vector field $\nu$ we consider its smooth completion $\tilde{X}$ and the resolution of singularities $\pi: \bar{X} \rightarrow \tilde{X}$ of the foliation induced by the vector field so that the resulting foliation $\mathcal{F}$ on $\bar{X}$ is reduced. Then $D=\pi^{-1}(\tilde{X} \backslash X)$ is the divisor at infinity and $E$ is the exceptional divisor of $\pi$ over $X$, i.e $\pi(E)=\Gamma$ is a finite subset of $X$. Moreover we have the following [13, p. 442].

7.1. Lemma. Each irreducible component of $D$ and each irreducible component of the exceptional divisor $E$ of $\pi: \bar{X} \rightarrow \tilde{X}$ are $\mathcal{F}$-invariant.

Following Brunella, consider now the MCQUILLAN's contraction $\tau: \bar{X} \rightarrow \bar{X}^{\prime}$ of a reduced foliation $\mathcal{F}$ on $\bar{X}$ to a nef reduced foliation $\mathcal{F}^{\prime}$ on $\bar{X}^{\prime}$ as a sequence of blowing downs

$$
\bar{X}=\bar{X}_{0} \rightarrow \bar{X}_{1} \rightarrow \ldots \rightarrow \bar{X}_{n-1} \rightarrow \bar{X}_{n}=\bar{X}^{\prime}
$$

of rational curves $F_{j} \subset \bar{X}_{j}$ as in Remark [5.2. Denote by $\mathcal{F}_{j}$ the foliation induced by $\mathcal{F}$ on $\bar{X}_{j}$, by $D_{j}$ and $E_{j}$ the images of $D$ and $E$ respectively. We call $F_{j}$ external if it is not contained in $D_{j} \cup E_{j}$. 
7.2. Lemma. (cf. [13, Lemma 6]) Let $F_{j}$ be external and let $\tilde{F}_{j}$ be the proper transform of $F_{j}$ in $\tilde{X}$. Then $\tilde{F}_{j} \cap X$ is an algebraic curve in $X \backslash \Gamma$ isomorphic to $\mathbb{C}$. Furthermore, for different external curves $F_{j}$ and $F_{i}$ the curves $\tilde{F}_{j} \cap X$ and $\tilde{F}_{i} \cap X$ are disjoint.

Proof. Note that $F_{j}$ must meet $D_{j}$ since otherwise its strict transform in $\bar{X}$ will be contained in $\pi^{-1}(X)$ but not in $E$ which is impossible for a complete curve. Then $D_{j} \cap F_{j}$ must be $\mathcal{F}_{j}$-invariant since both $D_{j}$ and $F_{j}$ are. Therefore, it is the only singular point of the foliation on $F_{j}$ (see Remark 5.2) ). By the same reason $F_{j}$ cannot meet $E_{j}$ which implies the first statement. To see the second one we note that $F_{j}$ and $F_{i}$ cannot meet outside $D$ since otherwise contrary to Remark $5.2 F_{j}$ has another singular point of the foliation besides $D_{j} \cap F_{j}$. Thus $\tilde{F}_{j} \cap X$ and $\tilde{F}_{i} \cap X$ are disjoint.

7.3. Remark. Let $L$ be the union $\bigcup \tilde{F}_{j}$ where $F_{j}$ runs over the set of external curves. By construction $L$ and therefore $X^{*}=X \backslash(L \cup \Gamma)$ are invariant with respect to the original foliation, i.e. the restriction of $\nu$ to $X^{*}$ is completely integrable.

Then BRunella considers morphism $r: Y \rightarrow \bar{X}^{\prime}$ as in Theorem 18 and the commutative diagram generated by it

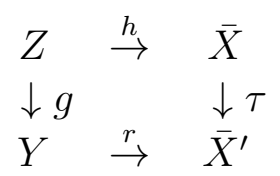

where $g: Z \rightarrow Y$ is a birational morphism and $h: Z \rightarrow \bar{X}$ is a ramified covering such that $r \circ g=\tau \circ h$. Set $R=D \cup E, R^{\prime}=\tau^{-1}(\tau(R))$, and $W=Z \backslash h^{-1}\left(R^{\prime}\right)$. Suppose that $\mu_{0}$ is the holomorphic vector field on projective variety $Y$ that generates fibration $\mathcal{G}$ from Theorem 18, Let $\mu$ be its lift to $Z$ via $g$.

7.4. Lemma. (cf. [13, Lemma 7]) Let $\mu, W, X^{*}, L$ be as before. Then

(1) the restriction of $h$ makes $W$ an unramified cover of $X^{*}$;

(2) the restriction $\left.g\right|_{W}: W \rightarrow Y$ is an embedding;

(3) elements of the flow associated with the restriction of $\left.\mu\right|_{W}$ are algebraic automorphisms of $W$.

Proof. By Theorem 18 morphism $r$ can be ramified only over singularities of $\bar{X}^{\prime}$. By construction these singularities are contained in $\tau(R)$. Thus $h$ can be ramified only over $R^{\prime}$. For (1) it remains to note that $R^{\prime}=D \cup E \cup L$.

Since $Z$ is the fibered product of $Y$ and $\bar{X}$ over $\bar{X}^{\prime}$ we see that $W$ is the fibered product of $Y$ and $\bar{X} \backslash R^{\prime}$ over $\bar{X}^{\prime}$. Hence for (2) it suffices to note that the restriction of $\tau$ to $\bar{X} \backslash R^{\prime}$ is an embedding.

We have also (3) since $\mu$ arises from a holomorphic vector field $\mu_{0}$ on the projective variety $Y$ and elements of its flow are algebraic automorphisms by Chow's theorem.

7.5. Corollary. Suppose that for any finite collection of disjoint lines in $X \backslash \Gamma$ and every finite unramified covering $W \rightarrow X \backslash(\Gamma \cup L)$ where $L$ is the union of these lines 
the manifold $W$ has a finite number of algebraic automorphisms. Then the case of $\operatorname{kod}(\mathcal{F})=0$ does not hold.

\section{Geometry of the surface $x+y+x y z=1$.}

Consider the hypersurface $S \subset \mathbb{C}_{x, y, z}^{3}$ given by $x+y+x y z=1$. It can be viewed as the affine modification of $\pi: S \rightarrow \mathbb{C}^{2}$ of $\mathbb{C}_{x, y}^{2}$ along the divisor $x y=0$ with center at $x y=1-x-y=0$ (i.e at points $(1,0)$ and $(0,1))$ since $z=(1-x-y) /(x y)$. Note that $S$ contains four lines $L_{1}, L_{2}, L_{3}$, and $L_{4}$ such that $\pi\left(L_{1}\right)=(0,1), \pi\left(L_{2}\right)=\{y=1\}$, $\pi\left(L_{3}\right)=(1,0)$, and $\pi\left(L_{4}\right)=\{x=1\}$.

8.1. Lemma. There is only one polynomial curve $L_{5}$ in $S$ different from $L_{1}, L_{2}, L_{3}, L_{4}$ and it is the proper transform of the line $x+y=1$ in $\mathbb{C}^{2}$.

Proof. Consider a non-constant morphism $\varphi: \mathbb{C} \rightarrow S$ whose image is not one of the four lines. Its composition with $\pi$ yields a morphism $\mathbb{C} \rightarrow \mathbb{C}^{2}$ given by $x=p(t)$ and $y=q(t)$ (where $p$ and $q$ are non-constant polynomials) whose image $C$ may meet the $x$-axis and the $y$-axis only at points $(1,0)$ and $(0,1)$ respectively. Furthermore, local branches of $C$ cannot be tangent to any of these axes since otherwise the proper transform of $C$ in $S$ (which is $\varphi(\mathbb{C})$ ) is a Riemann surfaces with at least two punctures contrary to the assumption that it is a polynomial curve. This implies that $1-p(t)$ is divisible by $q(t)$ while $1-q(t)$ is divisible by $p(t)$. In particular, $\operatorname{deg} p=\operatorname{deg} q$ and therefore, $p(t)=1-q(t)$. This yields the desired conclusion.

8.2. Remark. One can check that the equations of $L_{1}, L_{2}, L_{3}, L_{4}$, and $L_{5}$ in $S$ are $x=0, y z+1=0, y=0, x z+1=0$, and $z=0$ respectively.

8.3. Lemma. Let $\rho: S^{\prime} \rightarrow S$ be a finite morphism of smooth surfaces unramified over the complement to the five lines in $S$. Then $S^{\prime}$ has a finite number of algebraic automorphisms.

Proof. First note that for every polynomial curve $C$ in $S^{\prime}$ its image $\rho(C)$ is a polynomial curve in $S$. Thus the number of such curves in $S^{\prime}$ is finite. Any automorphism $\alpha$ of $S^{\prime}$ generates their permutation. Without loss of generality we can assume that this permutation is identical. Then the restriction of $\alpha$ yields an automorphism of the surface $H^{\prime} \subset S^{\prime}$ that is an unramified finite covering of the Kobayashi hyperbolic surface $H=S \backslash \bigcup_{i=1}^{5} L_{i}$ (and therefore $H^{\prime}$ is itself hyperbolic). There are at most finite number of such automorphisms and we have the desired conclusion.

8.4. Proposition. Let $\nu$ be a completely integrable algebraic vector field on $S$. Then

(1) either $\nu$ has a first integral which is a regular function with general fibers isomorphic to $\mathbb{C}^{*}$ or

(2) there is a regular function $P$ with general fibers isomorphic to $\mathbb{C}^{*}$ that is a Riccati fibration for $\nu$, i.e. the flow associated with $\nu$ transforms fibers of $P$ into fibers of $P$. 
Proof. We need to check conditions (i) and (ii) in Proposition 6.3. The first of them is always true because of Proposition 6.5. As for the second, the surface $S$ possesses an SNC-completion whose dual graph is a cycle consisting of three vertices of weights 1,0 , and 0 respectively. Thus Proposition 6.3 is applicable.

Assume now that $f$ is a rational first integral of $\nu$. Then general orbits of $\nu$ are contained in the fibers of $f$ on the complement $S^{*}$ to the set of indeterminacy points of $f$ in $S$. Since $S$ contains only five polynomial curves these fibers must be isomorphic to $\mathbb{C}^{*}$. Furthermore, since their closures must meet at indeterminacy points we see that such points cannot exist because otherwise the closures produce infinite number of polynomial curves. Thus $S^{*}=S$ and $f$ is regular which is (1).

In the absence of a rational first integral for $\nu$ let $\mathcal{F}$ be the associated foliation on an SNC-completion of $S$. By Corollary 7.5 and Lemma $8.3 \operatorname{kod}(\mathcal{F})$ cannot vanish, i.e. it is equal to 1 by Theorem [18, Now Proposition 6.2 implies (2).

\subsection{Lemma. The surface $S$ is factorial and its Euler characteristics is 2.}

Proof. Note that $S=T \cup L_{1} \cup L_{3}$ where $T=S \backslash\left(L_{1} \cup L_{3}\right)$ is a torus. Hence $\chi(S)=$ $\chi(T)+\chi\left(L_{1}\right)+\chi\left(L_{3}\right)=2$ by [23]. Then modification $\pi: S \rightarrow \mathbb{C}^{2}$ may be viewed as composition of two modifications $\pi^{\prime}: S \rightarrow S^{\prime}$ and $\pi^{\prime \prime}: S^{\prime} \rightarrow \mathbb{C}^{2}$ where $S^{\prime}$ is the affine modification of $\mathbb{C}^{2}$ along $x=0$ at center $(0,1)$ (i.e. $S^{\prime}$ is again isomorphic to $\mathbb{C}^{2}$ and therefore factorial) and $S$ is obtained as the affine modification along the proper transform of $y=0$ in $S^{\prime}$ (which is a $\mathbb{C}^{*}$-curve) with center at point $(1,0)$. That is, $S$ is obtained from $S^{\prime}$ by replacing an irreducible curve by a line $L_{3}$. The Nagata lemma (e.g., see [24, Lemma 19.20]) implies that $S$ remains factorial.

8.6. Proposition. Let $f$ be a regular function on $S$ whose general fibers are isomorphic to $\mathbb{C}^{*}$. Then up to a linear transformation $f$ coincides with one of the functions $x^{k} y^{l}$ (where $k$ and $l$ are relatively prime nonnegative integers with $k+l \geq 1$ ), $x z+1, y z+1$, and $z$.

Proof. Since the general fibers are isomorphic to $\mathbb{C}^{*}$ each connected component of singular fibers is either $\mathbb{C}^{*}$ or a curve of Euler characteristics one (more precisely either a line or a cross) by [78, Theorem 3]. Furthermore, there are exactly two such components because the Euler characteristics of $S$ is 2 by Lemma 8.5. They are disjoint and therefore the description of polynomials curves in $S$ implies that the union of these components contains one of the following pairs of lines: $\left(L_{1}, L_{3}\right),\left(L_{1}, L_{4}\right),\left(L_{2}, L_{3}\right),\left(L_{2}, L_{5}\right)$, and $\left(L_{4}, L_{5}\right)$. Consider the case of the first three pairs and tori $T_{13}=S \backslash\left(L_{1} \cup L_{3}\right)$, $T_{14}=S \backslash\left(L_{1} \cup L_{4}\right)$, and $T_{23}=S \backslash\left(L_{2} \cup L_{3}\right)$. The general fiber $F \simeq \mathbb{C}_{\xi}^{*}$ is contained in one of these tori, say $T_{13}$ that is isomorphic to $\mathbb{C}_{x}^{*} \times \mathbb{C}_{y}^{*}$. Hence up to constant factors $x=\xi^{k}$ and $y=\xi^{l}$ where $k$ and $l$ are coprime integers since the functions $x$ and $y$ separate points of $F$. Let $m$ and $n$ be integers such that $m k+n l=1$. Then $\xi=x^{m} y^{n}$ together with $f=x^{k} y^{-l}$ form a new coordinate system on $T$ such that the restriction 
of $f$ to $F$ is constant. Note that this $f$ is regular on $S$ only when the powers are nonnegative which yields the first functions mentioned in the formulation.

Note that $T_{14}$ is isomorphic to the torus $\mathbb{C}_{x}^{*} \times \mathbb{C}_{\zeta}^{*}$ where $\zeta=y /(x-1)$. Thus for $T_{14}$ this function $f$ must be of form $x^{k}(x-1)^{l} / y^{l}$. However for a nonzero $l$ this function is not regular on $S$ and we have to disregard it. Similarly in the case of $T_{23}$ we have to disregard function of the form $y^{k}(x-1)^{l} / x^{l}$.

Now consider the case when the zero fiber of $f$ contains $L_{4}$ and $L_{5}$ as components. Let us show that $f^{-1}(0)=L_{4} \cup L_{5}$. Indeed, assume to the contrary that this fiber contains another irreducible component $L$. Since $S$ is factorial by Lemma $8.5 L_{4}$ is given by zeros of $x z+1, L_{5}$ by zeros of $z$ (see Remark [8.2), and $L$ by zeros of another function $h$. All three functions are invertible on $T_{45}=S \backslash f^{-1}(0)$ and thus the group of invertible functions has at least three generators. This group is isomorphic to the group of integer first cohomology (e.g., see [35]) and therefore $H^{1}\left(T_{45}, \mathbb{Q}\right.$ ) is at least of rank 3. On the other hand nonzero fibers of $f$ do not contain connected components of Euler characteristics one and therefore $T_{45}$ is a $\mathbb{C}^{*}$-fibration over $\mathbb{C}^{*}$. By the technical Lemma 8.7 below its rational cohomology is at most of rank 2. This contradiction shows that $L$ cannot exist.

Since $f^{-1}(0)=L_{4} \cup L_{5}$ up to a constant factor we have $f=z^{k}(x z+1)^{l}$ where $k$ and $l$ are natural and, furthermore, relatively prime (since otherwise the general fiber of $f$ is not connected). Then taking into consideration the equation of $S$ we see that the equation of the projection $C$ of the fiber $f^{-1}(c)$ to $\mathbb{C}_{x, y}^{2}$ is given by

$$
(1-x-y)^{k}(1-x)^{l}=c x^{k} y^{k+l} \text {. }
$$

Hence over a neighborhood of $x=0$ (resp. $x=\infty$ ) up to constant factor the restriction of $y$ to $C$ behaves as $x^{-k /(k+l)}$ (resp. $x^{l /(k+l)}$ ). This implies that the projection of $C$ to the $x$-axis has ramification points of order $k+l$ over $x=0$ and $x=\infty$. Differentiating (8.1) with respect to $y$ we get

$$
-k(1-x-y)^{k-1}(1-x)^{l}=c(k+l) x^{k} y^{k+l-1} .
$$

Dividing (8.1) by this equality we get $1-x=\alpha y$ where $\alpha=l /(k+l)$. Plugging $\alpha y$ instead of $1-x$ into (8.1) we obtain a polynomial in $y$ of degree $2 k+l$ which yields additional ramification points. Since the projection of $C$ to the $x$-axis is $(k+l)$-sheeted the Riemann-Hurwitz formula implies that the genus of $C$ is positive which contradicts to the fact that $C$ is isomorphic to $\mathbb{C}^{*}$.

Now assume that $L_{4}$ and $L_{5}$ are contained in different fibers of $f$. Using again Lemma 8.7 below we can see that at least one of these two fibers is irreducible. That is, up to a linear transformation $f$ must coincide with either $z$ or $x z+1$.

By considering the pair $\left(L_{2}, L_{5}\right)$ instead of $\left(L_{4}, L_{5}\right)$ we get also $f=y z+1$ as a possibility which concludes the proof.

To make the proof of Proposition 8.6 complete we need the following. 
8.7. Lemma. Let $f: Y \rightarrow B$ be a $\mathbb{C}^{*}$-fibration (i.e. the general fibers of $f$ are isomorphic to $\mathbb{C}^{*}$ ) of a factorial affine algebraic surface over the base $B$ isomorphic to a line with $k$ deleted points. Then the dimension of $H_{1}(Y, \mathbb{Q})$ is at most $k+1$.

Proof. Since $Y$ is factorial the removal of an irreducible curve from $Y$ increases the number of generator of the group of regular invertible functions by 1 . Therefore, this procedure increases the dimension of the first rational homology by 1 (e.g., see [35]). Thus it suffices to prove the statement of Lemma in the case of $f$ being locally trivial since we can remove the singular fibers. If $Y$ is the direct product $B \times \mathbb{C}^{*}$ then $\operatorname{dim} H_{1}(Y, \mathbb{Q})=k+1$ by the Kunneth formula. In the general case the computation goes via the Leray spectral sequence but the dimension of homology can only decrease compared with the case of the direct product. This concludes the proof.

Theorem 19. Let $\nu$ be a completely integrable algebraic vector field on $S$. Then either (1) $\nu$ coincides with a vector field of form

$$
q(z)\left((1+x z) \frac{\partial}{\partial x}-(1+y z) \frac{\partial}{\partial y}\right)
$$

where $q$ is a polynomial or

(2) the restriction of $\nu$ to $T=S \backslash\left(L_{1} \cup L_{3}\right) \subset \mathbb{C}_{x, y}^{2}$ is a vector fields of form

$$
p\left(x^{k} y^{l}\right)\left(l x \frac{\partial}{\partial x}-k y \frac{\partial}{\partial y}\right)
$$

where $p$ is a polynomial with $p(0)=0$ and $k$ and $l$ are relatively prime nonnegative integers.

Proof. By Proposition 8.4 we have a regular function $P$ on $S$ with general fibers isomorphic to $\mathbb{C}^{*}$ such that $P$ is invariant with respect to the flow of $\nu$. All such possible $P^{\prime}$ 's are listed in Proposition 8.6. Suppose that $P=z$. Then this regular function has two singular fibers. Hence each individual fiber of $P$ is preserved by the flow, i.e. $P$ is the first integral. It remains to note that every field that has general fibers of $P$ as orbits is of form (1).

Now let $P=x^{k} y^{l}$ with natural relatively prime $k$ and $l$,

$$
\nu_{1}=x \partial / \partial x, \text { and } \nu_{2}=l x \partial / \partial x-k y \partial / \partial y
$$

Repeating the argument from [13, Proposition $2(2)$ ] we trivialize a neighborhood of a regular fiber of $P$, isomorphic to $\Delta \times \mathbb{C}_{\zeta}^{*}$, in such a way that $\nu_{1}$ is sent to $\partial / \partial \xi$ (where $\xi$ is a coordinate on the unit disc $\Delta$ ) and $\nu_{2}$ is sent to $\zeta \partial / \partial \zeta$. Extending the flow of $\nu$ to $\Delta \times \mathbb{C P}^{1}$ we find that $\nu$ is of form $\beta(\xi) \partial / \partial \xi+\alpha(\xi) \zeta \partial / \partial \zeta$ (because this extension must be tangent to the curves $\zeta=0$ and $\zeta=\infty$ ). Hence $\nu$ is of the form

$$
\nu=q\left(x^{k} y^{l}\right) \nu_{1}+p\left(x^{k} y^{l}\right) \nu_{2}
$$

where both functions $p$ and $q$ are regular on $\mathbb{C}_{\xi}^{*}$. However, if we want this rational field on $\mathbb{C}^{2}$ to be lifted regularly on $S$ we need to require that it vanishes at points $(1,0)$ 
and $(0,1) \in \mathbb{C}^{2}$. This implies that $p$ and consequently $q$ are polynomials on $\mathbb{C}_{\xi}$ and $p(0)=q(0)=0$. Furthermore, since $\nu$ is completely integrable $q$ must be constant and we have the desired form.

In the case of $P$ equal to $x z+1$ or $y z+1$ the same argument produces a field as in (2) but with the pair $(k, l)$ equal to $(1,0)$ or $(0,1)$ which concludes the proof.

8.8. Corollary. Every completely integrable algebraic vector field on $S$ has divergence zero with respect to the algebraic volume form $\omega=\frac{\mathrm{d} x}{x} \wedge \frac{\mathrm{d} y}{y}$. In particular, $S$ has no algebraic density property.

Proof. First note that the restriction of vector fields of type (2) from Theorem 19 to the torus $T=S \backslash\left(L_{1} \cup L_{3}\right)$ is completely integrable. Thus their divergence with respect to $\omega$ is zero [4]. It can be checked also by the direct computation which we do for the fields on type (1). We need the following two formulas (e.g., see [48])

$$
\operatorname{div}_{\omega}(f \nu)=f \operatorname{div}_{\omega}(\nu)+\nu(f) \text { and } \operatorname{div}_{f \omega}(\nu)=\operatorname{div}_{\omega}(\nu)+L_{\nu}(f) / f
$$

where $\nu$ is a vector field, $f$ is a regular function, and $L_{\nu}$ is the Lie derivative. In particular, $\operatorname{div}_{\omega}(f \nu)=0$ provided $\operatorname{div}_{\omega}(\nu)=0$ and $\nu(f)=0$. Thus for the fields of type (1) it suffices to consider $\nu=(1+x z) \frac{\partial}{\partial x}-(1+y z) \frac{\partial}{\partial y}$ because $z$ belongs to the kernel of $\nu$. Since $1+x z=(1-x) / y$ and $1+y z=(1-y) / x$ the restriction of $\nu$ to $T$ can be written in the from

$$
\frac{1-x}{y} \frac{\partial}{\partial x}-\frac{1-y}{x} \frac{\partial}{\partial y}
$$

The divergence of the first summand $\frac{1-x}{y} \frac{\partial}{\partial x}$ with respect to the standard form $\omega_{0}=$ $\mathrm{d} x \wedge \mathrm{d} y$ is $-1 / y$. Since $\omega=\frac{1}{x y} \omega_{0}$ its divergence with respect to $\omega$ is

$$
-1 / y+L_{\nu}\left(\frac{1}{x y}\right) /\left(\frac{1}{x y}\right)=-1 / y-(1-x) /(x y)=-1 /(x y) .
$$

Similarly, the divergence of the second summand $\frac{1-y}{x} \frac{\partial}{\partial y}$ is $-1 /(x y)$ and $\operatorname{div}_{\omega}(\nu)=0$ which implies the first statement.

Since $\operatorname{div}_{\omega}\left(\left[\nu_{1}, \nu_{2}\right]\right)=L_{\nu_{1}}\left(\operatorname{div}_{\omega}\left(\nu_{2}\right)\right)-L_{\nu_{2}}\left(\operatorname{div}_{\omega}\left(\nu_{1}\right)\right)$ (e.g., see [48]) the Lie algebra generated by completely integrable algebraic vector fields contains only fields of divergence zero with respect to $\omega$. Thus algebraic vector fields with nonzero divergence cannot be contained in this algebra which implies the absence of the algebraic density property.

It remains to show that the group of holomorphic automorphisms of $S$ generated by completely integrable algebraic vector fields is $m$-transitive for any natural $m$. For this consider the fields

$$
(1+x z) \frac{\partial}{\partial x}-(1+y z) \frac{\partial}{\partial y}, x y \frac{\partial}{\partial x}-(1+y z) \frac{\partial}{\partial z}, \text { and }
$$




$$
x y \frac{\partial}{\partial x}-(1+x z) \frac{\partial}{\partial z}
$$

on $S$. They are completely integrable fields from Theorem 19 (the first one is of type (1) and the last two are of type (2) with the pair $(k, l)$ equal to $(0,1)$ and $(1,0)$ respectively), they generate the tangent space of $S$ at each point, and their kernels in $\mathbb{C}[S]$ are $\mathbb{C}[z], \mathbb{C}[y]$, and $\mathbb{C}[x]$ respectively. Therefore, the desired conclusion follows from the next fact.

8.9. Proposition. Let $X$ be a smooth affine algebraic variety and $\sigma_{1}, \ldots, \sigma_{k}$ be completely integrable nonzero algebraic vector fields on $X$. Suppose that the kernel of $\sigma_{j}$ in $\mathbb{C}[X]$ is denoted by $\operatorname{Ker} \sigma_{j}$ and the following conditions hold

(i) $\sigma_{1}, \ldots, \sigma_{k}$ generate tangent space at any point of $X$;

(ii) there exist regular functions $f_{1} \in \operatorname{Ker} \sigma_{1}, \ldots, f_{k} \in \operatorname{Ker} \sigma_{k}$ such that each $f_{j}$ is not contained in $\bigcup_{l \neq j} \operatorname{Ker} \sigma_{l}$ and the $\operatorname{map}\left(f_{1}, \ldots, f_{k}\right): X \rightarrow \mathbb{C}^{k}$ is an embedding.

Then the group $G$ of holomorphic automorphisms generated by elements of the flows of completely integrable algebraic vector fields is $m$-transitive for every natural $m$.

Proof. Let us start with the following.

Claim. Let $X_{*}^{m}$ be the subset of points $\left(x_{1}, x_{2}, \ldots, x_{m}\right)$ in $X^{m}$ such that for any $j=1, \ldots, k$ we have

$$
f_{j}\left(x_{i}\right) \neq f_{j}\left(x_{l}\right)
$$

where $i \neq l$. Then its $G$-orbit $O \subset X^{m}$ contains all points of form $\left(x, x_{2}, \ldots, x_{m}\right)$ where $x$ runs over some neighborhood $U$ of $x_{1}$ in $X$.

Indeed, let $p_{j}(j=1, \ldots, n)$ be a polynomial in one variable such that the function $p_{j}\left(f_{j}\right)$ vanishes at all points $x_{2}, \ldots, x_{m}$ but not at $x_{1}$. Then the elements of flows of completely integrable vector fields $p_{1}\left(f_{1}\right) \sigma_{1}, \ldots, p_{k}\left(f_{k}\right) \sigma_{k}$ keeps points $x_{2}, \ldots, x_{n}$ fixed. On the other hand since these fields generate the tangent space $T_{x_{1}} X$ by (i), compositions of their exponents can send $x_{1}$ into any given point in a small neighborhood $U$ of $x_{1}$ which yields the Claim.

Note that $U$ can chosen so that it depends continuously on $\left(x_{1}, x_{2}, \ldots, x_{n}\right)$ in a neighborhood of this point in $X_{*}^{m}$, and we can also repeat this Claim with any $x_{j}$ instead of $x_{1}$. This implies immediately that $O \cap X_{*}^{m}$ is an open subset of $X_{*}^{m}$. Furthermore, it is closed in $X_{*}^{m}$ because if $\left(x_{1}^{\prime}, x_{2}^{\prime}, \ldots, x_{m}^{\prime}\right)$ belongs to its closure then the open set $O^{\prime} \cap X_{*}^{m}$ meets $O$ where $O^{\prime}$ is the $G$-orbit of $\left(x_{1}^{\prime}, x_{2}^{\prime}, \ldots, x_{m}^{\prime}\right)$. Thus $O$ contains $X_{*}^{m}$.

Now let $\left(x_{1}, x_{2}, \ldots, x_{m}\right) \in X^{m}$ be an $m$-tuple of distinct points that does not satisfy (8.2). Say $f_{j}\left(x_{1}\right)=f_{j}\left(x_{2}\right)$ for $j \leq n$ and $f_{i}\left(x_{1}\right) \neq f_{i}\left(x_{2}\right)$ for $n+1 \leq i \leq k$ (note that $n<k$ because of assumption (ii)). It suffices to show that a perturbation of $\left(x_{1}, x_{2}, \ldots, x_{m}\right)$ by an element of $G$ close to the identical automorphism destroys at least one of these equalities. Though a priori a perturbation may not destroy the equalities it enables us to assume that $\sigma_{k}$ is not tangent at $x_{1}$ to the fiber of $f_{1}$ (since otherwise $f_{1} \in \operatorname{Ker} \sigma_{k}$ contrary to (ii)). Choose again a polynomial $p_{k}$ such that $p_{k}\left(f_{k}\right)$ vanishes at $x_{2}$ but not at $x_{1}$. Then the flow of $p_{k}\left(f_{k}\right) \sigma_{k}$ changes the value of $f_{1}\left(x_{1}\right)$ while keeping the point $x_{2}$ fixed which concludes the proof. 


\section{REFERENCES}

[1] C. B. Aliprantis, K. C. Border, Infinite dimensional analysis. A hitchhiker's guide (third edition), Springer, Berlin, 2006, xxii+703 pp.

[2] E. Andersén, Volume-preserving automorphisms of $\mathbb{C}^{n}$, Complex Variables Theory Appl. 14 (1990), no. 1-4, 223-235.

[3] E. Andersén, L. Lempert, On the group of holomorphic automorphisms of $\mathbb{C}^{n}$, Invent. Math. 110 (1992), no. 2, 371-388.

[4] E. Andersén, Complete vector fields on $\left(\mathbb{C}^{*}\right)^{n}$, Proc. Amer. Math. Soc. 128 (2000), no. 4, 1079-1085.

[5] T. Asanuma, Non-linearizable algebraic $k^{*}$-actions on affine spaces, Invent. Math. 138 (1999), no. 2, 281-306.

[6] P. Bala, R. W. Carter, Classes of unipotent elements in simple algebraic groups. I. Math. Proc. Cambridge Philos. Soc. 79 (1976), no. 1, 401-425.

[7] P. Bala, R. W. Carter, Classes of unipotent elements in simple algebraic groups. II. Math. Proc. Cambridge Philos. Soc. 80 (1976), no. 1, 1-17.

[8] S. Borell, F. Kutzschebauch, Non-equivalent embeddings into complex Euclidean spaces. Internat. J. Math. 17 (2006), no. 9, 1033-1046.

[9] N. Bourbaki, Elements of Mathematics. Lie Groups and Lie Algebras. Chapters 7-9. Springer, Berlin-Heidelberg-New York, 2005.

[10] M. Brunella, Sur les courbes intgrales propres des champs de vecteurs polynomiaux, Topology 37 (1998), no. 6, 1229-1246.

[11] M. Brunella, Birational geometry of foliations, Monografas de Matemtica. [Mathematical Monographs] Instituto de Matemtica Pura e Aplicada (IMPA), Rio de Janeiro, 2000. 138 pp.

[12] M. Brunella, Foliations on complex projective surfaces, arXiv:math/0212082, (2002), 31 p.

[13] M. Brunella, Complete polynomial vector fields on the complex plane, Topology 43 (2004), 433-455.

[14] L. Bungart, Holomorphic functions with values in locally convex spaces and applications to integral formulas, Trans. Amer. Math. Soc. 111 (1964), 317-344.

[15] G. T. Buzzard, F. Forstnerič, A Carleman type theorem for proper holomorphic embeddings. Ark. Mat. 35 (1997), no. 1, 157-169.

[16] G. Buzzard, J. E. Fornæss, An embedding of $\mathbb{C}$ into $\mathbb{C}^{2}$ with hyperbolic complement, Math. Ann. 306 (1996), no. 3, p. 539-546.

[17] H. Derksen, F. Kutzschebauch, Nonlinearizable holomorphic group actions. Math. Ann. 311 (1998), no. 1, 41-53.

[18] H. Derksen, F. Kutzschebauch, Global holomorphic linearization of actions of compact Lie groups on $\mathbb{C}^{n}$, Contemporary Mathematics 222 (1999), p. 201-210.

[19] H. Derksen, F. Kutzschebauch, J. Winkelmann, Subvarieties of $C^{n}$ with non-extendable automorphisms. J. Reine Angew. Math. 508 (1999), 213-235.

[20] P. G. Dixon, J. Esterle, Michael's problem and the Poincaré-Fatou-Bieberbach phenomenon. Bull. Amer. Math. Soc. (N.S.) 15 (1986), no. 2, 127-187.

[21] F. Donzelli, A. Dvorsky, S. Kaliman, Algebraic density property of homogeneous spaces, arXiv:0806.1935, (2009), $26 \mathrm{p}$.

[22] J.M. Drezet, Luna's slice theorem and applications Algebraic group actions and quotients, 39-89, Hindawi Publ. Corp., Cairo, 2004. 
[23] A. Durfee, Algebraic varieties which are a disjoint union of subvarieties, Geometry and topology (Athens, Ga., 1985), 99-102, Lecture Notes in Pure and Appl. Math., 105, Dekker, New York, 1987.

[24] E. Eisenbud, Commutative algebra. With a view toward algebraic geometry, Graduate Texts in Mathematics, Springer-Verlag, New York, 1995, xvi+785 pp.

[25] Y. Eliashberg and M. Gromov, Embeddings of Stein manifolds of dimension $n$ into the affine space of dimension 3n/2 +1, Ann. of Math. (2), 136 (1992), no. 1, 123-135.

[26] W. Fulton, J. Harris, Representation Theory. A First Course Graduate Texts in Mathematics, 129, Readings in Mathematics, Springer-Verlag, New York, (1991).

[27] O. Forster, Plongements des variétés de Stein, Comm. Math. Helv., 45 (1970), p. 170-184.

[28] F. Forstnerič, B. Ivarsson, F. Kutzschebauch, J- Prezelj, An interpolation theorem for proper holomorphic embeddings. Math. Ann. 338 (2007), no. 3, 545-554.

[29] F. Forstnerič, J.-P. Rosay, Approximation of biholomorphic mappings by automorphisms of $\mathbb{C}^{n}$, Invent. Math. 112 (1993), no. 2, 323-349.

[30] F. Forstnerič, The Oka principle for sections of stratified fiber bundles Pure Appl. Math. Q. 6 (2010), no. 3, 843-874.

[31] F. Forstnerič, Equivalence of real submanifolds under volume-preserving holomorphic automorphisms of $C^{n}$. Duke Math. J. 77 (1995), no. 2, 431-445.

[32] F. Forstneric, E. Löw, Global holomorphic equivalence of smooth submanifolds in $C^{n}$. Indiana Univ. Math. J. 46 (1997), no. 1, 133-153.

[33] F. Forstneric, E. Löw, N. Øvrelid, Solving the $d$ - and $\bar{\partial}$-equations in thin tubes and applications to mappings. Michigan Math. J. 49 (2001), no. 2, 369-416.

[34] F. Forstnerič, J. Globevnik, J.-P. Rosay, Nonstraightenable complex lines in $\mathbb{C}^{2}$, Ark. Mat. 34 (1996), no. 1, 97-101.

[35] T. Fujita, On the topology of non-complete algebraic surfaces, J. Fac. Sci. Univ. Tokyo, Sect.IA, 29 (1982), 503-566.

[36] Ph. Griffiths, J. Harris, Principles of algebraic geometry, Pure and Applied Mathematics, Wiley-Interscience [John Wiley and Sons], New York, 1978. xii+813 pp.

[37] M. Gromov, Oka's principle for holomorphic sections of elliptic bundles, J. Amer. Math. Soc. 2 (1989), no. 4, 851-897.

[38] A. Grothendieck, On the de Rham cohomology of algebraic varieties, Inst. Hautes Etudes Sci. Publ. Math., 29 (1966), 95-105.

[39] R. Hartshorne, Algebraic Geometry, Springer-Verlag, New York Berlin Heidelberg London, 1977, $496 \mathrm{p}$.

[40] R. B. Holmes, Geometric functional analysis and its applications, Graduate Texts in Mathematics, 24, Springer-Verlag, New York-Heidelberg, 1975. x+246 pp.

[41] S. Kaliman, Extensions of isomorphisms between affine algebraic subvarieties of $k^{n}$ to automorphisms of $k^{n}$, Proc. Amer. Math. Soc. 113 (1991), no. 2, 325-334.

[42] S. Kaliman, Some facts about Eisenman intrinsic measures. Complex Variables Theory Appl. 27 (1995), no. 2, 163-173.

[43] S. Kaliman, M. Koras, L. Makar-Limanov, P. Russell, $\mathbf{C}^{*}$-actions on $\mathbf{C}^{3}$ are linearizable. Electron. Res. Announc. Amer. Math. Soc. 3 (1997), 63-71 (electronic).

[44] S. Kaliman, F. Kutzschebauch, Density property for hypersurfaces uv $=p(\bar{x})$, Math. Zeit. 258 (2008), 115-131.

[45] S. Kaliman, F. Kutzschebauch, Criteria for the density property of complex manifolds, Invent. Math. 172 (2008), 71-87.

[46] S. Kaliman, F. Kutzschebauch, Algebraic volume density property of affine algebraic manifolds, arXiv:0908.2416, (2009), 35p. 
[47] S. Kaliman, Actions of $\mathbb{C}^{*}$ and $\mathbb{C}_{+}$on affine algebraic varieties, Algebraic geometry - Seattle 2005. Part 2, 629-654, Proc. Sympos. Pure Math., 80, Part 2, Amer. Math. Soc., Providence, RI, 2009.

[48] S. Kobayashi, K. Nomizu, Foundations of differential geometry, Vol. I. Reprint of the 1969 original. Wiley Classics Library.

[49] F. Kutzschebauch, Andersén-Lempert-theory with parameters: a representation theoretic point of view. J. Algebra Appl.4 (2005), no. 3, 325-340.

[50] F. Kutzschebauch, Some results on embedding Stein spaces with interpolation. Ark. Mat. 43 (2005), no. 2, 419-425.

[51] F. Kutzschebauch, S. Lodin, Families of holomorphic embeddings and group actions on affine space, preprint (2009)

[52] F. Kutzschebauch, E. Löw, E. Fornæss Wold, Embedding some Riemann surfaces into $\mathbb{C}^{2}$ with interpolation. Math. Z. 262 (2009), no. 3, 603-611.

[53] J. M. Landsberg, L. Manivel, B. W. Westbury, Series of unipotent orbits. Experimental Mathematics, 13 (2004), 13-29.

[54] M. McQuillan, Non-commutative Mori Theory, IHES, M/01/42, (2001).

[55] H. Peters, E. Fornæss Wold, Non-autonomous basins of attraction and their boundaries. J. Geom. Anal. 15 (2005), no. 1, 123-136.

[56] R. Remmert, Sur les espaces analytiques holomorphiquement séparables et holomorphiquement convex, C. R. Acad. Sci. Paris 243 (1956), p. 118-121.

[57] J.-P. Rosay, Automorphisms of $\mathbb{C}^{n}$, a survey of Andersn-Lempert theory and applications, Complex geometric analysis in Pohang (1997), 131-145, Contemp. Math., 222, Amer. Math. Soc., Providence, RI, 1999.

[58] J.-P. Rosay, W. Rudin, Holomorphic maps from $\mathbb{C}^{n}$ to $\mathbb{C}^{n}$, Trans. Amer. Math. Soc. 310 (1988), no. 1, p. 47-86.

[59] J.-P. Rosay, W. Rudin, Holomorphic embeddings of $\mathbb{C}$ in $\mathbb{C}^{n}$, in: Several Complex Variables, Math. Notes 38, Proceedings of the Mittag-Leffler Institute 1987-88, Princeton University Press (1993), p. 563-569.

[60] J. Schürmann, Embeddings of Stein spaces into affine spaces of minimal dimension, Math. Ann. 307 (1997), no. 3, p. 381-399.

[61] L. Schwartz, Produits tensoriels topologiques et espaces nuclaires, Séminaire Inst. Henri Poincaré, 1953/1954.

[62] L. Schwartz, Thorie des distributions valeurs vectorielles. I (French), Ann. Inst. Fourier, Grenoble 7 (1957), 1-141.

[63] V. Srinivas, On the embedding dimension of an affine variety, Math. Ann. 289 (1991), no. 1, $125-132$.

[64] B. Stensönes, Fatou-Bieberbach domains with $C^{\infty}$-smooth boundary. Ann. of Math. (2) 145 (1997), no. 2, 365-377.

[65] M. Suzuki, Sur les oprations holomorphes du groupe additif complexe sur l'espace de deux variables complexes, Ann. Sci. cole Norm. Sup. 10 (1977), no. 4, 517-546.

[66] M. Suzuki, Sur les oprations holomorphes de $\mathbb{C}$ et de $\mathbb{C}^{*}$ sur un espace de Stein, Fonctions de plusieurs variables complexes, III (Sm. Franois Norguet, 1975-1977), pp. 80-88, 394, Lecture Notes in Math., 670, Springer, Berlin, 1978.

[67] A. Toth, D. Varolin, Holomorphic diffeomorphisms of complex semisimple Lie groups, Invent. Math. 139 (2000), no. 2, 351-369.

[68] A. Toth, D. Varolin, Holomorphic diffeomorphisms of semisimple homogenous spaces, Compos. Math. 142 (2006), no. 5, 1308-1326.

[69] F. Trèves, Topological vector spaces, distributions and kernels, Academic Press, New YorkLondon, 1967, xvi+624 pp. 
[70] D. Varolin, The density property for complex manifolds and geometric structures, J. Geom. Anal. 11 (2001), no. 1, 135-160.

[71] D. Varolin, The density property for complex manifolds and geometric structures. II, Internat. J. Math. 11 (2000), no. 6, 837-847.

[72] J. Winkelmann, On automorphisms of complements of analytic subsets in $C^{n}$, Math. Z. 204 (1990), no. 1, 117-127.

[73] J. Winkelmann, Large discrete sets in Stein manifolds Math. Z. 236 (2001), no. 4, 883-901.

[74] E. Fornæss Wold, A Fatou-Bieberbach domain in $\mathbb{C}^{2}$ which is not Runge. Math. Ann. 340 (2008), no. 4, 775-780.

[75] E. Fornæss Wold, Embedding subsets of tori properly into $\mathbb{C}^{2}$. Ann. Inst. Fourier (Grenoble) 57 (2007), no. 5, 1537-1555.

[76] E. Fornæss Wold, Embedding Riemann surfaces properly into $\mathbb{C}^{2}$. Internat. J. Math. 17 (2006), no. $8,963-974$.

[77] E. Fornæss Wold, Proper holomorphic embeddings of finitely and some infinitely connected subsets of $\mathbb{C}$ into $\mathbb{C}^{2}$. Math. Z. 252 (2006), no. 1, 1-9.

[78] M. G. Zaidenberg, Isotrivial families of curves on affine surfaces, and the characterization of the affine plane (Russian) Izv. Akad. Nauk SSSR Ser. Mat. 51 (1987), no. 3, 534-567, 688; translation in Math. USSR-Izv. 30 (1988), no. 3, 503-532.

Department of Mathematics, University of Miami, Coral Gables, FL 33124, USA

E-mail address: kaliman@math.miami.edu

Department of Mathematics, University of Bern, Bern, Switzerland,

E-mail address: frank.kutzschebauch@math.unibe.ch 